\title{
Strong interactions between highly-dynamic lamina-associated domains and the nuclear envelope stabilize the 3D architecture of Drosophila interphase chromatin
}

\author{
Igor S Tolokh ${ }^{1}$, Nicholas Allen Kinney ${ }^{1,2,3}$, Igor V Sharakhov ${ }^{2 *}$ and Alexey $\bigvee$ Onufriev ${ }^{1,4,5 *}$
}

\footnotetext{
${ }^{*}$ Correspondence: igor@vt.edu; alexey@cs.vt.edu

${ }^{2}$ Department of Entomology, Virginia Tech, 24061, Blacksburg, VA, USA

${ }^{1}$ Department of Computer Science, Virginia Tech, 24061 Blacksburg, VA, USA

Full list of author information is available at the end of the article
}

\begin{abstract}
Background: Interactions between topologically associating domains (TADs), and between the nuclear envelope (NE) and lamina-associated domains (LADs) are expected to shape various aspects of 3D chromatin structure and dynamics at the level of the entire nucleus; however, a full quantitative picture is still lacking. Relevant genome-wide experiments that may provide statistically significant conclusions remain difficult.

Results: We have developed a coarse-grained dynamic model of the Drosophila melanogaster nucleus at TAD $(\sim 100 \mathrm{~kb})$ resolution that explicitly accounts for four distinct epigenetic classes of TADs and describes time evolution of the chromatin over the entire interphase. Best agreement with experiment is achieved when the simulation includes an entire biological system - an ensemble of complete nuclei, corresponding to the experimentally observed set of mutual arrangements of chromosomes, properly weighted according to experiment. The model is validated against multiple experiments, including those that describe changes in chromatin induced by lamin depletion.

Predicted positioning of LADs at the NE is highly dynamic (mobile) - the same LAD can attach, detach, and re-attach itself to the NE multiple times during interphase. The average probability of a LAD to be found at the NE varies by an order of magnitude, determined by the highly variable local density of nearby LADs along the genome. The distribution of LADs along the genome has a strong effect on the average radial positioning of individual TADs, playing a notable role in maintaining a non-random average global structure of chromatin.

Predicted sensitivity of fruit fly chromatin structure to the balance between TAD-TAD and LAD-NE interactions, also observed previously in models of mammalian chromosomes, suggests conservation of this principle of chromatin organisation across species of higher eukaryotes. Reduction of LAD-NE affinity weakly affects local chromatin structure, as seen in the model-derived $\mathrm{Hi}-\mathrm{C}$ maps, however, its wild-type strength substantially reduces sensitivity of the chromatin density distribution to variations in the strength of TAD-TAD interactions.

Conclusions: A dynamical model of the entire 3D fruit fly genome makes multiple genome-wide predictions of biological interest. We conjecture that one important role of LADs and their attractive interactions with the NE is to create the average global chromatin structure and stabilize it against inevitable cell-to-cell variations of TAD-TAD interactions.
\end{abstract}

Keywords: Drosophila; Hi-C; chromosome model; nuclear envelope 


\section{Introduction}

Chromosomes are intricately folded and packaged inside the cell nuclei where they interact with nuclear bodies and remodeling factors [1, 2]. This complex three-dimensional (3D) organization of the genome plays a crucial role in regulation of gene expression, DNA replication, and DNA double-strand break repair $[3,4,5,6,7,8,9]$. Topologically associating domains (TADs) have emerged as the fundamental structural and functional units of chromatin organization in the eukaryotic cell nuclei, from fruit fly to human $[1,10,11]$. Interactions between TADs contribute to higher level, hierarchical genome organization [12]: multiple TADs within each compartment and multiple compartments within each chromosome territory. Boundaries between individual TADs are conserved among cell types and sometimes across species $[13,14,15,16,10,17,18]$; this feature makes TAD a natural structural unit of chromatin useful for modeling purposes and reasoning [19, 20], including in this work. TADs interact among themselves forming dynamic, relatively short-living 3D-structures, that segregate chromatin into mutually excluded active (type A) and inactive (type B) compartments [21, 12]. Averaged over time of the entire interphase, the effect of TAD-TAD interactions is seen in experimental Hi-C maps course-gained to TAD resolution $[1,13,22]$. However, such Hi-C maps do not reveal how dynamic (mobile) are the 3D positions of each individual TAD. Live-cell imaging studies of interphase nuclei in different organisms showed that some chromatin loci are mobile $[23,24,25,26]$. Also, studies suggested that constrains on the motion of chromatin loci can result from their interaction with structural elements such as the nuclear lamina (NL) which lines the inner surface of the nuclear envelope (NE), or the nuclear pore complexes [25, 27, 28, 29, 30]. However, genome-wide analysis of nuclear dynamics of all "freely floating" and "tethered" chromatin loci has not been performed. DamID experiments have identified hundreds of genome regions that "anchor" to the NL $[31,32,33,34,35]$. These genome regions that have specific affinity to the NL are called lamina-associated domains (LADs) [36, 37, 38]. Single-cell DamID studies demonstrate [39, 40] that LADs identified in a human cell population may in fact be located either at the nuclear periphery (30\% of LADs) or in the nuclear interior in individual cells [39]. The LADs that contacted the NL were shown to move during interphase [39], but this movement is confined to a relatively narrow layer next to the NE. Positioning of LADs in mammalian cells $[39,40,38,37,41]$ was shown to be stochastic: averaged across cells, a certain $\%$ of LADs is bound to the NE in a given cell, but the binding of specific LADs varies from cell to cell. These observations raise several questions. Is positioning of LADs only stochastic between cells (i.e., LADs have mostly stable positions within a cell, but highly variable positions between cells) or is it truly dynamic/mobile in each cell (i.e., individual LADs can change their positions relative to the NE significantly within a cell during interphase). If LADs are mobile, on what time-scale? Also, do different LADs have different probabilities of being attached to the lamina?

States of transcription activation and repression are also linked to the positioning of chromatin with respect to the NE. LADs are typically repressive environments. This supports the notion that the nuclear periphery is generally occupied by inactive chromatin [42]. TADs and LADs are similar in size: each is approximately $1 \mathrm{Mb}$ in the human genome $[13,32]$ and approximately $90-100 \mathrm{~kb}$ in the fruit fly genome 
$[1,43,36]$. For example, there are 1169 TADs and 412 LADs in the Drosophila melanogaster genome $[1,36]$. The role of LAD-NE interactions and its interplay with compartmentalization of TADs has been explored in fruit flies [44] and mammals [45, 46, 41, 47, 48, 49]. For example, depletion of lamins from Drosophila S2 cells leads to chromatin compaction and reduction in spatial segregation of the chromatin into active and inactive compartments [44]. Simulations of multiple copies of mouse chromosomes 1 and 2 [45] have disproved the role of lamina as the main driver of compartmentalization [37]. At the same time, attractions between heterochromatic TADs emerged as the main force of compartmentalization, while LAD-NE interactions are crucial for controlling the global spatial morphology of the nucleus [45]. However, a number of questions remains unanswered, related to sensitivity of the general organization principles with respect to global loss of chromatin-lamina interactions, which may occur in disease or senescence [41]. Given that attractive TAD-TAD interactions play a major role in compartmentalization, will the global chromatin architecture become more sensitive or less sensitive to TAD-TAD interactions upon lamin depletion? Will the cell-to-cell variability in the 3D chromatin organization increase or decrease upon disruption of LAD-NE interactions?

Understanding the principles and factors leading to the formation of non-random $3 \mathrm{D}$ genome organization and, ultimately, to the structure-function relationships in chromatin is prerequisite to understanding cell physiology [2, 50, 45]. However, relevant experiments that may provide statistically significant conclusions remain difficult. Computational models that faithfully reproduce available experimental data are indispensable for the 3D genome reconstruction problem (3D-GRP) based on DNA-proximity ligation data such as $5 \mathrm{C}, \mathrm{Hi}-\mathrm{C}$ and Pore-C, and they can generate valuable predictions and guide experiment $[51,52,53,54,55,56,57,45,41,58$, $19,59,60,61,62,63,64,65,66,46,67,68,69,70,71,72,73]$. A particularly strong feature of computational models is that they can answer questions that may be very hard to address experimentally. For example, tracing the movement of a given TAD or a few chromosomal loci upon detachment from the NE caused by a lamin depletion is possible experimentally [44, 74], but making statistically significant statements based on 1000s of TADs in the nucleus would be extremely laborious. Another example is obtaining time-resolved Hi-C maps for a synchronized cell population: possible, but difficult experimentally, with only a handful of studies so far $[75,76]$. Models can generate testable hypotheses, the most promising of which can be checked experimentally.

Since simulating an entire eukaryotic genome on biologically meaningful timescales at fully atomistic resolution is still far out of reach [77], current practical models accept various levels of approximation determined by the balance of research goals and computational feasibility. A large class of recent computational models that aim to understand 3D structure of chromatin employ the "beads-on-a-string" coarse-graining approach $[48,41,78,65,53,79,56]$ borrowed from polymer physics $[80,81]$. For complex organisms, such as mammals, various further approximations are often made to reach the desired temporal or/and spatial resolution, such as considering only a small subset [45] of the dozens of the original chromosomes or even only one chromosome $[41,46,47]$. In that respect, modeling of "simpler" nuclei $[60,67]$, in particular of higher eukaryotes, e.g., that of the well-studied fruit fly, 
with its only five major chromosome arms offers a computational advantage that may translate into an ability to model the entire nucleus $[19,56,58]$, which, in turn, may help to answer questions otherwise difficult to address. Consensus conclusions about chromatin organization that emerge from using models of different types applied to substantially different organisms are of value, as these conclusions hint at conservation of general principles across species.

Our work contributes to developing a quantitative understanding of various aspects of 3D organization of chromatin by constructing and employing a computational model, trained and tested on multiple experimental data sets describing the 3D organization of chromatin in D. melanogaster. We believe that it offers a number of novel insights, both biological and methodological, while also reinforcing robustness of some of the conclusions about chromatin organization previously made using models of mammalian nuclei.

\section{Methods}

Background and rationale for choosing model building blocks at TAD level

In D. melanogaster nuclei each TAD compartmentalizes $\sim 100 \mathrm{~kb}$ of chromatin [1]; there are four major classes of TADs: Active, Null, PcG and HP1 [1] (see Fig. S1 in the SI). Active TADs are defined by histone $\mathrm{H} 3$ modifications such as trimethylation of lysine 4 and 36 (H3K4me3 and H3K36me3). PcG TADs are enriched in Polycomb group proteins and histone mark H3K27me3. HP1 TADs are associated with classical heterochromatin marks such as H3K9me2 histone modification, heterochromatin proteins HP1 and $\mathrm{Su}($ var)3-9. Null TADs lack known specific chromatin marks [1].

The four classes of TADs in fruit fly refine the distinction between type A and type B compartments. Type A compartments are organized by Active class TADs and defined by early replication, proteins, and histone modifications involved in active transcription. Type B compartments are defined by late replication and modifications that silence genes. These compartments consist of transcriptionally silent TADs (PcG, HP1 and Null) and occupy a larger portion of the genome than type A compartments $[1,43]$.

\section{Model: chromosome representation}

We consider $D$. melanogaster female interphase nuclei with a diploid set of four chromosomes $(2,3,4$ and $\mathrm{X})$. Hi-C data suggest [1] that D. melanogaster genome (excluding pericentromeric constitutive heterochromatin (HET) and centromeric (CEN) regions) is organized into 1169 TADs. Since the same TADs in homologous chromosomes are almost always in close proximity to each other [82, 83, 19, 84, 85], we represent each pair of homologous TADs by a single spherical bead. In addition to these 1169 TAD-beads, we introduce 4 beads representing CEN regions and 6 beads representing HET domains.

Using the "beads-on-a-string" model [80, 86], four chains of the beads represent paired homologous chromosomes (Chr). In Chr 2 and 3, the L- and R-arms are connected via three-bead HET-CEN-HET structure. Chr 4 begins from a CEN-HET bead pair, and Chr X ends with a HET-CEN two-bead structure. The Chr chains are placed inside of a spherical boundary (see Fig. 1, top panel) which represents the NE. We also consider a nucleolus which is modeled as a spherical bead of 0.333 $\mu \mathrm{m}$ radius placed at half distance between the nucleus center and the NE. 
bioRxiv preprint doi: https://doi.org/10.1101/2022.01.28.478236; this version posted January 28, 2022. The copyright holder for this preprint (which was not certified by peer review) is the author/funder, who has granted bioRxiv a license to display the preprint in perpetuity. It is made Tolokh et al. available under aCC-BY-NC 4.0 International license.

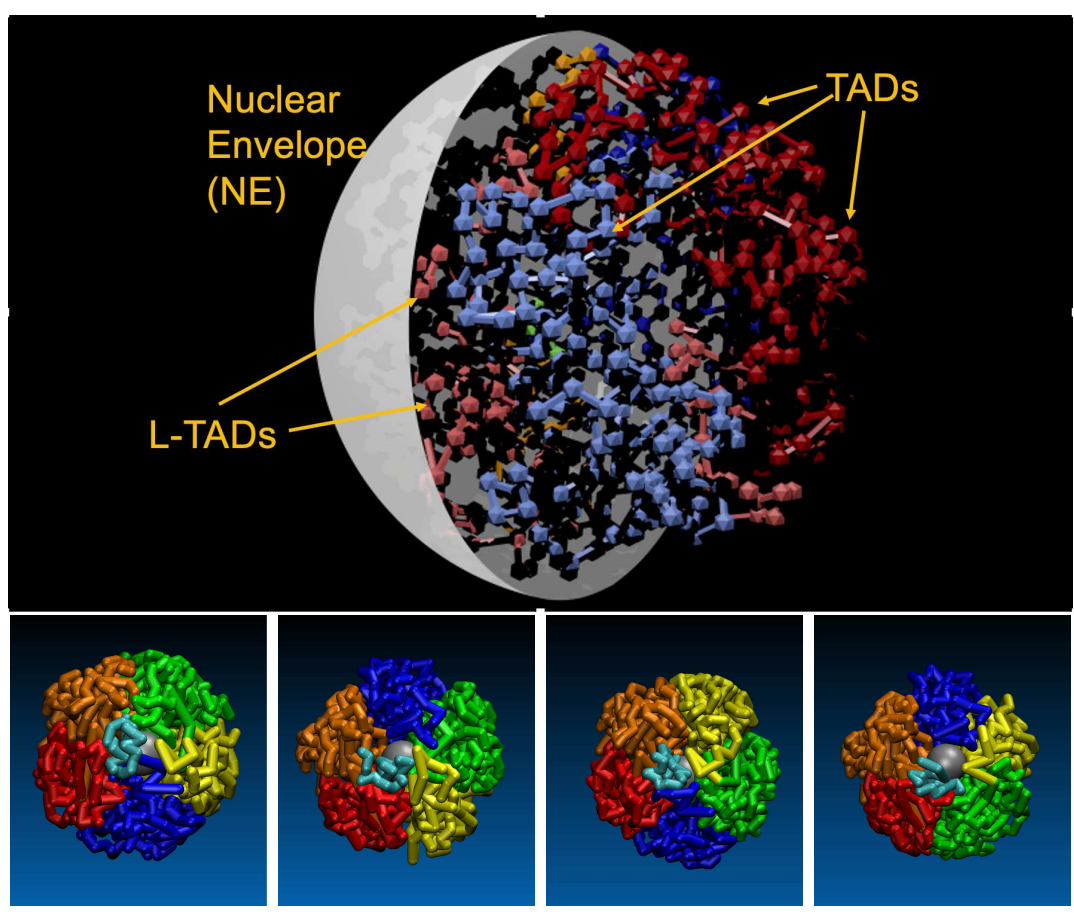

Figure 1 A schematic of our model (top) and the four initial configurations (bottom) of the D. melanogaster chromosome arms (2L (red), 2R (orange), 3L (yellow), 3R (green), $\mathrm{X}$ (blue) and 4 (cyan)) that serve as the starting points of the simulations described here. The nucleolus is shown as a grey sphere (bottom panel) The arms are fully "territorial" at the beginning of each simulation. The initial configurations correspond to different the mutual arrangements of the chromosome arms (nucleus topologies) experimentally determined by Hochstrasser et al. [87]. (From left to right): CIS-X6S, CIS-X7N, TRANS-X3S and TRANS-X4N nucleus topologies. Here, the "CIS" configuration refers to chromosome arrangement in which the two " $\mathrm{R}$ " arms (orange and green) or two "L" arms (red and yellow) of autosomes are next to each other in 3D space, while "TRANS" arrangement is complementary to it.

\section{Bead size and mass}

See detailed description in the SI.

Bead-bead interactions and bead types

See detailed description in the SI.

Interactions in 268 specific pairs of remote loci

See detailed description in the SI.

Arrangement of chromosome arms (nucleus topology) and their initial configurations Several mutual arrangements of the chromosome chains that correspond to CIS and TRANS relative arrangements of the Chr 2 and 3 arms, and two most probable positions ("North"-"N" and "South"-"S") of the Chr X have been observed experimentally [87] (see Fig. 1, bottom panels). The CIS arrangement refers to the one in which the R-arms (or the L-arms) of the autosomes are next to each other. The TRANS arrangement refers to the positions of the R- and L-arms of the autosomes next to each other. In the simulations, we use the following arrangements of the nucleus topology [87]: CIS-X6S, CIS-X7N, TRANS-X3S and TRANS-X4N. The 
CIS:TRANS arrangements ratio is assigned its experimental weight of 2 , meaning that twice more replicas of CIS topologies are simulated. In addition, each of these 6 properly weighted arrangements has 3 replicas to account for 3 different nucleus sizes used. Thus, the entire simulated ensemble consists of 18 model nuclei.

To create initial configurations of the bead chains that represent L- and R-arms of Chrs 2 and 3, and Chr X, we generated them as separate linear strings of beads parallel to z-axis (the axis of centromere-to-telomere chromosome polarization). Each chain is initially separately restrained by two planes intersecting along the zaxis with the dihedral angle $72^{\circ}$. A harmonic interaction between the nucleus center and each bead is applied using Eq. S1 (see the SI) with the effective bond length 0.99 $\mu \mathrm{m}$ and harmonic spring parameter $\kappa=0.5 k T$. A subsequent Langevin dynamics of the restrained chains over $1 \times 10^{5} 1.36 \mathrm{~ns}$ time steps produced collapsed fractallike Chr-arm configurations [86]. The L- and R-arms are then connected through the CEN beads, Chr 4 is added at the "North" pole, and large restraining sphere is placed around the chromosomes. A set of short Langevin dynamics simulations with a decreasing radius of the restraining sphere brings the size of the model nuclei to their specified final values. The resulting configurations are presented in Fig. 1.

Nuclear envelope and its interactions with chromatin chains

We model the NE as a spherical boundary that restrains the motion of the chromosomes and can attract LADs [33, 36, 37, 38]. Following [56], we map positions of 412 LADs [36] (a median size of LADs is about $90 \mathrm{~kb}$ ) onto the chains of 1169 TADs. If TAD contains LAD, then the corresponding bead can attractively interact with the NE. After mapping, we have determined 350 TADs that contain LADs (LTADs). We describe all L-TAD-NE (LAD-NE) attractive interactions by the LJ-cos potential (Eq. S2, see the SI) with a single well depth parameter $\epsilon_{L}$.

Fraction of LADs at the NE is calculated as the average (over the described above ensemble of 18 nuclei) fraction of L-TAD beads within (position of bead centers) 0.09 $\mu \mathrm{m}$ layer (average bead radius) at the NE. The thickness of this layer corresponds to about $0.2 \mu \mathrm{m}$ layer of beads which are in contact with the NE.

Interactions of the NE with TADs not containing LADs are described by the purely repulsive potential (see Eq. S3 and a more detailed description in the SI).

\section{Lamin Mutant model}

The Lamin mutant nucleus model has the L-TAD-NE affinity parameter $\epsilon_{L}$ reduced to $0.1 k T$ level. All other interaction parameters are unchanged from the wild-type (WT) model. (See a more detailed description in the SI).

\section{Experimental chromatin density profiles}

The results of the simulations are compared with the experimental chromatin density data [88], briefly described in the SI.

\section{Simulation of chromatin dynamics}

Langevin dynamics simulations have been performed using ESPResSo 3.3.1 package [89] by solving the Langevin equation of motion:

$$
m_{i} \ddot{\mathbf{r}_{i}}=-\nabla_{i} U-\gamma m_{i} \dot{\mathbf{r}}_{i}+\mathbf{L}_{i}(t)
$$


Here, $\mathbf{r}_{i}$ is the position of bead $i$ with mass $m_{i}, U$ is the potential energy of the system. The last two terms describe the interaction with the solvent: a velocity dependent friction force, characterised by the parameter $\gamma$, and a random Gaussian white noise force $\mathbf{L}_{i}$. The integration time step is $t_{\text {step }}=0.01 \tau$, where $\tau=\sigma_{\min } \sqrt{m_{\min } / \epsilon}$ is the LJ time scale [81, 90]. Using $\epsilon=3 k T$ we obtain $\tau=136 \mathrm{~ns}$ and, accordingly, $t_{\text {step }}=1.36 \mathrm{~ns} /$ which was used for all our simulations. For the simulations used to narrow down the range of the interactions parameters, we generated $40 \times 10^{6}$ time step trajectories with $\gamma=1 / \tau$. For the production simulations with the selected $\mathrm{WT}$ and Lamin mutant interaction parameter sets, we generated $400 \times 10^{6}$ time steps trajectories using $\gamma=0.01 / \tau$. This much smaller $\gamma$ allows one to effectively speed up the simulations ( $\sim 20$ times), which is one of the key benefits [91] of the implicit solvent approach used here. Also, in this regime of small friction the bead inertia and, accordingly, the difference in bead masses may become important.

\section{Simulation timescale}

To relate the simulation timescale with the experiment [92], that is to map the simulation time onto real biological time, we compared [56] a diffusive motion of model beads with the experimental interphase chromatin diffusion, and used the match to estimate the scaling factor $\lambda$ that converts the simulation time to real biological time. We calculated time dependencies of distance $R_{i}(t)$ between bead $i$ and the nucleus center for 9 randomly selected beads, which do not contain LADs. The dependence of the mean squared displacement (MSD) on the time interval $\Delta t$, $\left\langle\Delta R_{i}^{2}(\Delta t)\right\rangle=\left\langle\left[R_{i}(t+\Delta t)-R_{i}(t)\right]^{2}\right\rangle$, was calculated for 3 different values of the friction parameter $\gamma(0.01 / \tau, 0.1 / \tau$ and $1 / \tau)$. The averaging was performed over 9 selected beads along the 18 trajectories. We fit the first $300 \cdot 10^{3}$ time steps of each of the three curves $\left\langle\Delta R_{i}^{2}(\Delta t)\right\rangle$ (see Fig. S3 in the SI) with the following equation for sub-diffusive motion of chromosomal loci [92]:

$$
\left\langle\Delta R_{i}^{2}(\Delta t)\right\rangle=4 D_{\text {app }}(\Delta t / \lambda)^{0.39}
$$

where $D_{a p p}$ is the apparent diffusion coefficient. This equation (with $\lambda=1$ and $\Delta t$ in seconds) describes the experimentally observed diffusion of chromosomal loci over the time periods ranging from 1 to $10^{3} \mathrm{~s}$ [92]. We obtain reasonable fits of the simulation derived MSDs with $4 D_{a p p}=0.061 \mu \mathrm{m}^{2}$ and $\lambda=10^{4}$ for $\gamma=0.01 / \tau$, $\lambda=2.8 \cdot 10^{4}$ for $\gamma=0.1 / \tau$, and $\lambda=20 \cdot 10^{4}$ for $\gamma=1 / \tau$ (for $\Delta t$ in the time steps). These values of $\lambda$ give us the number of simulation time steps that corresponds to 1 sec of a real biological time. The scaling translates the $40 \times 10^{6}$ time step simulations with $\gamma=1 / \tau$ into $3 \mathrm{~min}$ of real nucleus time, and long production $400 \times 10^{6}$ time step simulations with $\gamma=0.01 / \tau$ as corresponding to $667 \mathrm{~min}(11 \mathrm{~h})$ of real time.

\section{Contact probability $(\mathrm{Hi}-\mathrm{C})$ map}

The TAD-TAD contact probability map (Hi-C map) of the model is defined as the average of Hi-C maps (1169x1169) calculated over 18 trajectories of the ensemble of 18 model nuclei of different sizes and mutual arrangements of the chromosomes. (See a more detailed description in the SI). 


\section{Model development}

Our model consists of 1179 "soft" beads that represent homologous pairs of TADs (1169 beads), pericentromeric constitutive heterochromatin domains (6 HET beads) and centromeric chromatin domains (4 CEN beads) (see "Methods"). The beads representing TADs are split into four types which correspond to four major epigenetic TAD classes: Active, Null, PcG and HP1 [1]. The beads are combined into four chromosome chains (Chrs 2, 3, 4 and X) using "beads-on-a-string" model $[78,65,53,79]$. Nucleolus is modeled as an additional constrained bead.

The nuclear envelope (NE), a spherical boundary surrounding the chromosome chains (see Fig. 1), constrains the motion of the beads within the nucleus by repulsive interactions. At the same time, the NE can attract L-TADs (TADs that contain LADs) [31, 36]) representing LAD-NE interactions.

Compared to the previous fruit fly interphase chromatin model [56], our model, Refs. [45, 41, 46, 93] for mammalian nuclei, utilizes the attractive interactions between non-bonded beads. We assume that this presumably protein mediated TADTAD attraction $[94,95]$ is a TAD-class dependent [93], since different combinations of proteins are bound to different epigenetic classes of TADs [96] with their characteristic histone modifications.

Experimentally observed compartmentalization of chromatin [21] into active euchromatin (type A compartments) and more densely packed inactive heterochromatin (type B compartments) allows us to assume [45, 93] at least three types of TAD-TAD interactions: A-A, B-B and A-B. To allow the compartmentalization, some general relations (the so-called Flory-Huggins rule [97]) between these three types of TAD-TAD interactions have to be considered.

We also introduce a set of "specific" non-bonded TAD-TAD interactions for 268 TAD (bead) pairs which form "long-range" contacts with the increased probabilities [1]. These attractive interactions are described by a set of effective potentials with the well depths determined from the experimental contact probabilities for the corresponding TAD pairs (see "Methods" in the SI).

\section{Interaction parameters of the models}

The attractive protein mediated interactions between TADs can spread over a several $k T$ range of energies [98, 45].

To determine "optimum" bead-bead and L-TAD-NE interaction parameters of the model $D$. melanogaster interphase nuclei we use simultaneously these three major criteria (selection rules):

(1) Maximum possible Pearson's correlation coefficient between model derived TADTAD contact probability map (model Hi-C map) and the experimental, wild-type Hi-C map [1], reduced to the TAD-TAD contact probabilities [19]);

(2) The fraction of LADs (LAD containing beads, L-TADs) at the NE matches the experiment for the WT nuclei: $25 \%$ [31]);

(3) The commonly used condition for the chromatin compartmentalization is satisfied. This is the so-called Flory-Huggins rule [97]) - the strength of the interactions between different (A and $\mathrm{B}$ ) unit types should satisfy the phase separation criterion: interaction $\mathrm{A}-\mathrm{B}<(\mathrm{A}-\mathrm{A}+\mathrm{B}-\mathrm{B}) / 2$.

Three stages of model development are used (see the description in the SI). 
The final optimum WT set of the interaction parameters is shown in the second column of the Table 1 below. We use this set for 10x longer simulations reported in the next section. The resulting chromatin density profiles will be compared with the available experimental data [88]. We will also investigate how sensitive is the chromatin structure to the deviations in the parameters.

\section{The resulting model}

The main capabilities of the model are illustrated in Fig. 2, where we present the snapshots of the temporal evolution of the nucleus with the TRANS-X3S nucleus topology along one of the production trajectories, corresponding to about $11 \mathrm{hrs}$ of biological time. The initial $t=0$ full ensemble averaged Hi-C map and the difference maps relative to $t=0$ are presented as well. The snapshots and the maps show the expected small decay [56] of the "perfect" original chromosome territories and local structures, while also demonstrating that reasonably distinct territories still exist 11 hours into the interphase, providing the first "sanity check" of our model.

\section{Results}

Dynamic model of chromosomes in fruit-fly at TAD resolution.

We have developed a coarse-grained "beads-on-a-string" model of D. melanogaster female interphase nuclei at TAD resolution (the average TAD size is $\sim 100 \mathrm{~kb}$ ), where a bead represents a pair of homologous TADs in paired homologous chromosomes. Four main types of beads corresponding to four major epigenetic classes of TADs [1] - Active, Null, PcG and HP1 - are used. The arrangements of the chromosome chains (nucleus topologies) in the model nuclei correspond to the experimentally observed CIS and TRANS mutual arrangements of the L and R chromosome arms and the position of X chromosome [87], see Fig. 1 in "Methods".

The model is trained to agree with the experimental Hi-C map, Fig. 3 (top panel) $[1,19]$, and lamin-DamID data [31] for the WT nuclei, and to satisfy the general polymer physics restrictions imposed on the strengths of the attractive interactions between beads of different types. The model derived TAD-TAD contact probability maps, i.e., model Hi-C maps, have been calculated for the ensemble of "young" nuclei, corresponding to about $\sim 3$ min of real biological time ( $40 \times 10^{6}$ time steps).

Unless otherwise specified, the results are averages over the ensemble of all different chromosome topologies and nucleus sizes (18 systems in total, see "Methods"). The selected WT parameter set provides a reasonably accurate reproduction of the experimental Hi-C map (Pearson's correlation coefficient is 0.956).

The model is validated against several different experiments not used for its training (see below) and is capable of predicting the evolution of the 3D chromatin architecture (see Fig. 2) on time scales up to 11 hours, enough to approximate the entire interphase of fruit fly nucleus [26]. The Hi-C map calculated using long " $11 \mathrm{~h}$ " trajectories for the WT parameter set has Pearson's correlation coefficient with the experimental Hi-C map 0.954. This value suggests that reducing friction coefficient $\gamma$ to 0.01 of its original value used for the model parameters development does not affect much of the local chromatin structure. A small decrease is expected due to time evolution and decay of the initial configurations. 


\section{WT nucleus vs. Lamin mutant nucleus}

To assess the role of the NE in organization of the chromatin architecture, the Lamin depleted nucleus model has been created by reducing the LAD-NE affinity from relatively strong $4 k T$ WT value to essentially zero, see "Methods". The rest of the model parameters were kept the same.

Two model derived WT and Lamin mutant radial TAD density distributions are shown in Fig. 4 (top panel). These distributions are in reasonable agreement with recently reported experimental chromatin density distributions [88] shown in Fig. 4 (bottom panel). The model faithfully reproduces some non-trivial nuances of the experimental distribution, including the "flatness" of the of density profile away from the NE for Lamin mutant, as opposed to, e.g., continued growth of the density toward the center of the nucleus that can occur if the model parameters deviate from their optimal values, see below.

This agreement provides a strong validation to the model, independent of the experimental data used for its training.

Comparing the WT and Lamin mutant chromatin distributions, Fig. 4, one can see a substantial shift of the chromatin density toward the NE in the WT nuclei. Due to LAD-NE attractive interactions in WT nuclei, a more compact globule-like distribution in the Lamin mutants, with a very low chromatin density near the $\mathrm{NE}$, is transformed into a more extended distribution in the WT nuclei, with a chromatin density peak near the NE and substantially reduced chromatin density in the central nucleus region. The effects of increased chromatin compaction and its detachment from the NE upon reduction of the LAD-NE attraction strength were also observed in the experiments with Drosophila S2 cells [44] and in the models of single human chromosomes [41,47]. Relocation of LADs to the nuclear interior has been observed in the model of single human chromosome [46], as well as in the model of several mouse chromosomes [45].

Calculations of the TAD-TAD contact probability (Hi-C) maps and their comparisons with the available experimental Hi-C data [1] (see Fig. 3) have been one of the criteria for selecting the interaction parameters for the WT nucleus model. Comparing the model derived " $11 \mathrm{~h}$ " Hi-C maps for the Lamin mutant and WT nuclei (Fig. 5), one can see that the changes in the contact probabilities are in agreement with the expected changes in the chromatin density distribution upon transition to the Lamin mutant seen in Fig. 4. A more compact and dense chromatin in the Lamin mutant has slightly higher TAD-TAD contact probabilities both within the chromosome arms and between the arms. Also, the specific TAD-TAD contacts produce more intensive spots on the Lamin mutant map (Fig. 5) compared to the Hi-C map for the WT model (Fig. 3, bottom panel). The model also reproduces key qualitative result from Ref. [44] that lamin depletion enhances interactions between active and inactive chromatin, impairing spatial segregation of active and inactive compartments. Our model provides a quantitative estimate for the increase of contacts of Null TADs with Active TADs in the Lamin mutant relative to the WT (see the sums of the selected contact probabilities for each Null TAD with Active TADs in Fig. S9 in the SI). The averaged relative increase is $22 \%$. This is yet another validation of the model, independent of the data sets used to build the model. 
NE as an "attractive enclosure"

Comparing the model derived Lamin mutant and WT chromatin distributions (Fig. 4, top panel) one can see that the main consequence of the almost complete elimination of the LAD-NE affinity is a significant "global" change in the chromatin density profile. The peak of the chromatin density in the WT nuclei at about 1.9 $\mu \mathrm{m}$ from the nucleus center (at about $94 \%$ of the relative distance from the center) disappears in the Lamin mutant, and the chromatin is shifted away from the NE. The TAD density in the nearest to the NE layer of TADs drops 6 times compared to the WT value, while the density in the central region of the nucleus doubles, making the resulting chromatin distribution more compact. Such changes suggest that main mechanical role of the NE is not mere confinement of chromatin, but, rather, the $\mathrm{NE}$ acts as an "attractive enclosure", which redistributes the chromatin, shifting most of it from the interior to the nuclear periphery. A similar general conclusion about this role of the NE was reached in the context of models of single human chromosomes interacting with the NE [41, 47]. We note that the respective parameter regimes that lead to this common conclusion, e.g., the ratios of TAD-TAD to LAD-NE interaction strengths, may be quite different from ours.

What we have found unexpected is how quickly - on the minute time-scale the compact chromatin organization deteriorates once the confining NE is removed completely, see Fig. S5 in the SI. Possible biological implications of this finding are touched upon in "Discussion".

\section{D chromatin architecture is highly sensitive to small variations in the interactions of} its key elements.

To investigate how different interaction types affect the chromatin properties we have simulated the nuclei with the interaction parameters deviating from the WT and the corresponding Lamin mutant set values.

\section{Effect of LAD-NE affinity variation}

One of the question which clarifies the role of the NE is how the deviation of the LAD-NE affinity from its WT value $(4 k T)$ affects the chromatin distribution? In Fig. S6 (top panel), the radial distributions of TADs in the model at different levels of LAD-NE affinity (from $0.1 k T$ (Lamin mutant) to $5 k T$ ) are presented. One can see that the increase of LAD-NE affinity by $0.5 k T$ from its WT value leads to a substantial $(\sim 2 \mathrm{x})$ decrease of the TAD density in the central nucleus region and, at the same time, to a $20 \%$ increase of the chromatin density peak at the NE. A more interesting behaviour can be observed when LAD-NE affinity is decreased. The $0.5 k T$ decrease of the affinity results in a substantial reduction of the density peak value relative to the chromatin density in the central nucleus region (from 1.8 to 1.1). A further decrease of the affinity to $3 \mathrm{kT}$, which is only a $25 \%$ change, leads to a complete transformation of the chromatin density profile to the Lamin mutant-like profile: the density peak at the NE disappears, a substantial amount of the chromatin moves away from the NE, the interior density increases by $80 \%$ compared to its WT value. The summary of these effects is presented in Table 1.

This strong dependence of the chromatin density profile on the LAD-NE affinity suggests that both the number of LADs in the fruit fly genome and their affinity to the NE are likely "tuned" by Nature to be rather close to values corresponding to a transition between the WT-like and Lamin mutant-like chromatin distributions. 
bioRxiv preprint doi: https://doi.org/10.1101/2022.01.28.478236; this version posted January 28, 2022. The copyright holder for this preprint (which was not certified by peer review) is the author/funder, who has granted bioRxiv a license to display the preprint in perpetuity. It is made

Table 1 Effects of Changes in TAD Interaction Parameters on Chromatin (CHR) Distribution and Chromosome Arms contacts. Single "+" ("-") denotes a small increase (decrease) in the corresponding contact probability. "++" ("- -") denotes a moderate increase (decrease), while "+++" denotes a significant increase.

\begin{tabular}{|c|c|c|c|c|}
\hline $\begin{array}{l}\text { Interaction } \\
\text { type }\end{array}$ & $\begin{array}{l}\text { WT } \\
\text { value }\end{array}$ & $\begin{array}{l}\text { Change } \\
\text { from WT }\end{array}$ & $\begin{array}{l}\text { Effect on distributions of Chromatin (CHR) } \\
\text { and LADs (L-TADs) }\end{array}$ & $\begin{array}{l}\text { Intra- and Inter-Arm } \\
\text { contacts changes }\end{array}$ \\
\hline $\begin{array}{l}\text { L-TAD-NE } \\
\text { (LAD-NE) } \\
\text { affinity }\end{array}$ & $4 \mathrm{kT}$ & $\begin{array}{l}+1 \mathrm{kT} \\
-1 \mathrm{kT}\end{array}$ & $\begin{array}{l}60 \% \text { increase of LAD fraction at NE (from } 0.25 \text { to } 0.4 \text { ) } \\
46 \% \text { increase of CHR density peak at NE (Fig.S6, Top) } \\
60 \% \text { decrease of LAD fraction at NE (from } 0.25 \text { to } 0.1 \text { ) } \\
43 \% \text { decrease of CHR density at WT peak position, } \\
\text { peak disappears (Fig. S6, Top) }\end{array}$ & $\begin{array}{l}\text { Intra }(+/--), \text { Inter }(-) \\
\text { Intra }(++/-), \text { Inter }(+)\end{array}$ \\
\hline Null-Null & $1.5 \mathrm{kT}$ & $\begin{array}{l}+1 \mathrm{kT} \\
-1.4 \mathrm{kT}\end{array}$ & $\begin{array}{l}36 \% \text { decrease of LAD fraction at NE (from } 0.25 \text { to } 0.16 \text { ), } \\
0.6 \mu \mathrm{m} \text { shift of CHR density peak from NE (Fig.S6, Bot) } \\
\text { Minor changes in CHR density profile }\end{array}$ & $\begin{array}{c}\text { Intra }(+++), \text { Inter }(++) \\
\text { Intra }(--), \text { Inter }(-)\end{array}$ \\
\hline PcG-PcG & $1.5 \mathrm{kT}$ & $-1.4 \mathrm{kT}$ & Minor changes in CHR density profile & Intra $(+/--)$, Inter $(-)$ \\
\hline HP1-HP1 & $1.5 \mathrm{kT}$ & & & \\
\hline Active-Act. & $0.1 \mathrm{kT}$ & $+0.4 \mathrm{kT}$ & No change in CHR density profile & Intra $(+/-)$, Inter $(+/-)$ \\
\hline Cross-type & $0.5 \mathrm{kT}$ & $+0.5 \mathrm{kT}$ & WT: minor change of CHR density profile (Fig. 6) & Intra $(+++), \ln t e r(++)$ \\
\hline
\end{tabular}

Effects of TAD-TAD interaction variation

We already described in "Methods" how we selected the values of the TAD-TAD interaction parameters which produce a good agreement with the experimental HiC map [1, 19] and LAD distribution [31]. Here we will discuss what effects the deviations from those optimal parameters will have on the chromatin distribution and the TAD-TAD contact probabilities. The summary of these effects is presented in Table 1.

Active-Active TAD interactions: Small increase (from 0.1 to $0.5 \mathrm{kT}$ ) does not lead to a noticeable change in the TAD radial distribution. Small variations in both directions of intra-arm and inter-arm TAD-TAD contact probabilities are observed.

Null-Null TAD interactions: Decreasing the amplitude of these interactions from $1.5 k T$ (WT value) to $0.1 k T$ produces a small effect on the radial chromatin distribution - a $20 \%$ increase of the chromatin density in the central nucleus region, and a small decrease of the density near the peak at the nucleus periphery, Fig. S6 (bottom panel). Similar effect of partial chromatin redistribution toward the nuclear center upon reduction of the attraction between B-type beads has been observed in the single chromosome model of chromatin described in Ref. [41]. This small redistribution of the chromatin in our model results in a minor decrease of intraarm and inter-arm TAD-TAD contact intensities. On the other hand, the increase of the Null-Null interactions by $1 k T$ (to $2.5 \mathrm{kT}$ ) leads to a substantial shift of the chromatin from the NE decreasing the fraction of L-TADs at the NE from $25 \%$ to $16 \%$ by transforming a more narrow main peak near the NE at $1.5-1.9$ $\mu \mathrm{m}$ into a wider peak at $0.9-1.7 \mu \mathrm{m}$, Fig. S6 (bottom panel). Similar effect of the shift of chromatin density toward the central region upon increase of the mutual attraction between B-type beads, when some of them have affinity to the NE, has been observed in the one-chromosome chromatin model described in Ref. [47]. At the same time, the effect predicted in Ref. [41] is the opposite, at least at the same ratio of LAD-NE to B-B attractions as used in our model.

The shift of the chromatin density peak in our model when increasing the NullNull attraction to $2.5 \mathrm{kT}$ (see Fig. S6 in the SI (bottom panel, blue line)) is accompanied by a noticeable increase of intra-arm and inter-arm TAD-TAD contacts probabilities with a reduction of Pearson's correlation coefficient with the experimental Hi-C map from 0.956 to 0.934 . Since 228 of 492 Null TADs contain LADs 
(the most enriched in LADs class of TADs, see Fig. S1 in the SI), these changes suggest a competition between the LAD-NE and Null-Null (TAD-TAD) interactions for the "optimal" chromatin structure. For comparison, only 54 of 494 Active TADs contain LADs, and the other two less numerous TAD classes contain even smaller numbers of L-TADs (50 of 131 PcG TADs and 18 of 52 HP1 TADs). This suggests that the competition between their TAD-TAD and LAD-NE interactions seems to be less important in the formation of the radial chromatin structure.

PcG-PcG TAD interactions: As in the case of Null-Null interactions, the decrease (from 1.5 to $0.1 \mathrm{kT}$ ) of the attraction between PcG TADs results in minor changes in the chromatin distribution and TAD-TAD contact probabilities. Due to the relative paucity of the PcG TADs we did not pursue further analysis of their selective influence on the chromatin structure.

Cross-type TAD-TAD interactions in Lamin mutant vs. WT: Does the WT level of LAD-NE affinity make chromatin density profile robust to changes in strength of cross-type TAD-TAD interactions? Decreasing the cross-type interactions from $0.5 k T$ (WT value) to $0.1 k T$ produces practically no change in the WT nuclei and minor changes in the Lamin mutant chromatin distribution $(10 \%-15 \%$ density decrease in the central regions due to small expansion of the chromatin toward the NE), Fig. 6. A small decrease of intra-arm and inter-arm TAD-TAD contacts is observed in both WT and Lamin mutant nuclei. On the other hand, increasing these interactions by $0.5 \mathrm{kT}$ (to $1.0 \mathrm{kT}$ ) results in a substantial (80\%$100 \%$ ) increase of the chromatin density in the central regions of the Lamin mutant due to further compaction of the chromatin in the globule-like state. The same change of the interactions in the WT nuclei leads to only a small increase of the density (about 20\%) in the central regions, Fig. 6. A significant increase of intraarm TAD-TAD contacts and a moderate increase of inter-arm TAD-TAD contacts are observed in both WT and Lamin mutant nuclei, as it can be seen on the Hi-C map differences (see Fig. S7 in the SI).

The substantial chromatin density changes in the Lamin mutant and the small corresponding changes in the WT nuclei demonstrate that in the absence of the attractive LAD-NE interactions the chromatin distribution is very sensitive to the small changes in the cross-type TAD-TAD interactions. The reduction of this sensitivity in the presence of LAD-NE interactions suggests a stabilizing role of these interactions in maintaining native chromatin distribution in the WT nuclei.

Role of LAD-NE interactions in positioning of TADs relative to NE

To investigate the dynamic positioning of individual TADs within the nucleus relative to the NE, and the role of LAD-NE interactions in this positioning, we have partitioned the nucleus into two spherically symmetric compartments of equal volume: the central spherical region of $1.6 \mu \mathrm{m}$ radius, and the spherical layer $(0.4 \mu \mathrm{m}$ thickness) adjacent to the NE. In the WT nuclei this $0.4 \mu \mathrm{m}$ layer contains about $70 \%$ of all L-TADs. The probabilities of individual TADs to be in that adjacent to the NE layer for the WT and Lamin mutant nuclei are shown in Fig. 7.

One can see that in the WT nuclei the TADs are partitioned into two major groups: TADs that have high $(0.5-0.95)$ probability to be in the $0.4 \mu \mathrm{m}$ layer near the NE (outer half the nuclear volume), and the ones that tend to stay away from 
the NE, with the probability in the range $0.1-0.5$ to be in that "near NE" layer. The TADs in this second group are in the sections of the chromosomes containing relatively long continuous stretches (10-16 TADs) without LADs. Quantitatively, one can characterize linear L-TAD density along the chromosome chain (L-TAD frequency of occurrence), $f_{L}$, as the ratio of the numbers of L-TADs to the number of all TADs in a given chromosome section. We see a clear correlation between the probability $\left(p_{N E}\right)$ of TAD to be in the $0.4 \mu \mathrm{m}$ "near NE" layer and the $f_{L}$ value, which can be compared to the average $f_{L}$ value across the whole genome, 0.30 . For example, the 1-st dip $\left(p_{N E}=0.13\right.$, Fig. 7$)$, in the probability distribution for the 2L-arm TADs from \#69 to \#72 is in the stretch of 23 TADs with only a single L-TAD, having the L-TAD density $f_{L}=0.08$. This density is almost 4 times lower that the genome-average value $f_{L}=0.30$. Another examples of the regions in the second group (low $p_{N E}$ ): in 2R-arm - TADs from \#301 to \#400 $\left(p_{N E}<0.34\right)$ have the average L-TAD density $f_{L}=0.19$; in $3 \mathrm{R}$-arm - TADs from \#711 to \#733 $\left(p_{N E}<0.34\right)$ have the average $f_{L}=0.13$. On the other hand, the regions in the first group (high $p_{N E}$ ) have a noticeably higher than average L-TAD density: in 2L-arm - TADs from \#155 to \#168 $\left(p_{N E}>0.8\right)$ have the average $f_{L}=0.59$; in X chromosome - TADs from \#1097 to \#1112 $\left(p_{N E}>0.68\right)$ have the average L-TAD density $f_{L}=0.47$.

This stable separation of the average radial positions of different TADs suggests that the chromatin, despite being in a liquid-like state, has some "averaged global structure" determined by the distribution of LADs (L-TADs) along the chromosomes.

In the Lamin mutant nuclei, the probabilities of TADs to stay closer to the NE (in the $0.4 \mu \mathrm{m}$ "near NE" layer), presented in Fig. 7, are in the range 0.1-0.4. The overall reduction of these probabilities compared to the WT values, reflecting the change of the TAD's average radial positions, is in agreement with the detachment of the chromatin from the NE and its compactization in the Lamin mutant nuclei seen on the chromatin density distributions, Fig. 4.

LADs in fruit fly are highly dynamic (mobile)

It is well-known that the subset of LADs found at the NE differs substantially from cell to cell. Here we ask if LADs in an individual fruit fly nucleus may also be mobile, and if so, to what extent.

Experimentally, there are 412 LADs in D. melanogaster nucleus [31, 36]; they are approximately evenly distributed along the three largest chromosomes: X, 2 and 3. As discussed in "Methods", we have mapped these LADs onto the 1169 TADs $[1,19]$ and have found LADs in 350 TADs (L-TADs). The WT value of the LADNE affinity ( $4 k T$ ) leads to the $25 \%$ fraction of L-TADs being, on average, in the nearest to the NE layer. Increasing or decreasing the LAD-NE affinity relative to its WT value in our simulations leads to an increase or a decrease of this fraction, see Fig. S4 in the SI. This sensitivity suggests a dynamic balance between the number of L-TADs attached to the NE at any given moment and the rest of L-TADs. That is, L-TADs can frequently attach to and detach from the NE during the interphase and are not permanently anchored to the NE in any given cell. Visualization of motion of randomly selected L-TADs in each chromosome shows that they are indeed highly 
mobile on time-scale of 20 minutes, see Fig. S8 in the SI, and the corresponding movie. One can see that, unlike human LADs [38, 37, 39], fruit fly LADs can quickly move though a significant portion of the nuclear volume.

To confirm the suggestion about a dynamic nature of LAD binding to the NE in the WT nuclei, we have computed probabilities of L-TADs to be (the center of L-TAD bead) within a very thin, $0.2 \mu$ m layer immediately adjacent to the NE (see Fig. 8). The thickness of this layer roughly correspond to the average diameter of beads in our model, which means that TADs in this layer can be considered in contact with the NE. To distinguish between cell-to-cell stochasticity and LAD mobility within individual cells, the probabilities are computed both for a single nucleus with CIS-X6S nucleus topology and for the ensemble of 6 nuclei with 4 different CIS and TRANS nucleus topologies. All nuclei are of the same size $(2 \mu \mathrm{m}$ radius).

First, we note that our model agrees with experiment for two L-TAD regions, for which their behavior in the Lamin depleted vs. WT fruit fly nuclei is known [44] (see the caption of Fig. 7 for details). From our genome-wide model prediction for all of the L-TADs, the probabilities to be in the $0.2 \mu \mathrm{m}$ layer at the NE are lower than 0.85 . The fact that the probabilities computed for a single nucleus are noticeably less than 1 supports the hypothesis that during the interphase all LTADs (LADs) bind to and unbind from the NE, within one and the same nucleus, see also Fig. S8 in the SI, and the corresponding movie. Even those L-TADs with the highest level of NE-binding probability are not permanently attached to the NE. Another interesting observation is the rather wide spread of the L-TAD binding probabilities seen in Fig. 8. Despite the fact that the model makes a simplification by considering all of the LAD-NE affinities to be the same (neglecting the variations in LAD size), we still predict very different levels of L-TAD binding to the NE. We suggest that this large difference in binding - the L-TAD probability to be in the 0.2 $\mu \mathrm{m}$ layer at the NE varies from 0.07 to 0.84 - is mostly related to the very different linear L-TAD density, $f_{L}$, in the chromosome regions surrounding these L-TADs. For example, the $f_{L}$ varies from 0.08 for L-TAD \#78 to 0.7 for L-TAD \#1015, with the corresponding probabilities to be found at the NE being 0.08 and 0.84 , respectively. Note that both $f_{L}$ values are significantly different from the average linear L-TAD density for all four chromosomes, which is 0.30 .

To further explore the predicted dynamic nature of L-TAD binding to the NE, we have computed radial distributions of the positions of two L-TADs, PcG L-TAD \#435 and Null L-TAD \#120, in the WT and Lamin mutant nuclei, see Fig. 9 (top and middle panels). One can see that for the WT nuclei, where L-TADs have 4 $k T$ affinity to the NE, the distributions are bi-modal: they have a bound mode, characterized by a large narrow density peak at the NE, and a diffusive mode with a noticeable density in the nucleus interior. In the case of the Null L-TAD \#120 (Fig. 9, middle panel), the WT diffusive mode has a greater amplitude, close to the average single TAD density in the nucleus $\left(\sim 0.03 \mu \mathrm{m}^{-3}\right)$. In the case of the PcG L-TAD \#435 (Fig. 9, top panel), the WT diffusive mode has a much smaller amplitude $\left(\sim 0.01 \mu \mathrm{m}^{-3}\right)$ with more of the TAD position density localized in the bound mode. These bi-modal LAD distributions demonstrate further, that in the WT nuclei, LADs are not permanently bound to the NE, but are instead rather 
mobile, being able to attach and detach during the interphase. Similar bi-modal distributions of LADs have been observed in a polymer model of a region of human chromosome $5[46]$.

In addition to L-TADs, in the WT nuclei the shift of the radial positioning toward the NE is also observed for most other TADs (see Fig. 7). A typical behaviour of radial distributions of these TADs is demonstrated using as an example the distribution for the Active TAD \#22, depicted in Fig. 9 (bottom panel). The wide distribution peak in the Lamin mutant nuclei is shifted toward the NE in the WT nuclei, which is in agreement with different probabilities of this TAD to be within the $0.4 \mu \mathrm{m}$ layer near the NE (half the nuclear volume) for the Lamin mutant and WT nuclei: 0.30 and 0.62 , respectively.

An interesting exception related to this general chromatin density shift toward the NE in the WT nuclei, compared to the Lamin depleted mutant, are a small groups of TADs (e.g., TADs with IDs in the ranges $64-80$ and $312-371$ in $2 \mathrm{~L}$ and $2 \mathrm{R}$ chromosome arms) that demonstrate small shifts in the radial density distributions toward the NE in the Lamin mutant. We suggest that this opposite pattern is related to the very low linear LAD density and, accordingly, very low averaged attraction to the NE of these select regions of chromosomes, relative to the neighbouring regions with higher than average LAD densities. In the WT nuclei, due to the competition among TADs for the limited volume near the NE, these special groups of TADs with a very low linear LAD density and low average attraction to the NE are effectively pushed away from the NE. Consistent with the above explanation, the differences in the average attraction towards the NE are eliminated in the Lamin mutant nuclei, leading to a much smaller spread of TAD probabilities to be in the "near NE" 0.4 $\mu \mathrm{m}$ layer. The remaining small variation - upturns in the green trace at the ends of each chromosome arm in Fig. 7 - is consistent with a greater probability to be at the nuclear periphery for the centromeric and telomeric regions of chromosomes.

The distributions of radial TAD positioning discussed above further suggest that the TADs are highly dynamic in both WT and Lamin mutant interphase nuclei.

\section{Discussion}

This work has two types of outcomes: methodological advances and biological predictions. The latter are focused on the role of interactions between structural components of chromatin such as TADs, as well as their interactions with the NE, in determining the local chromatin structure and 3D global architecture and their stability.

\section{Methodological Advances}

We have developed a novel coarse-grained "beads-on-a-string" model of chromatin of the entire D. melanogaster interphase nucleus at TAD resolution, $\sim 100 \mathrm{~kb}$ per TAD. The physics-based, as opposed to purely data-driven, approach allows us to answer many "what if" questions, including the effect of the chromosome-NE interactions on chromatin architecture, which is the subject of many recent experimental studies [44, 88, 73, 45, 99, 100, 29, 101]. Compared to many existing physics-based models of chromatin architecture in higher eukaryotes, our approach has several methodological novelties; these make a tangible difference with respect to biologically relevant predictions the model can make. 
First and foremost, we are simulating an entire biological system - an ensemble of nuclei, corresponding to the experimentally observed set of mutual spatial arrangements of chromosome arm (CIS and TRANS), properly weighted according to experiment. As we have shown, considering the entire biological system within a physics-based model proves important to reproducing experiment, specifically better agreement with the experimental Hi-C map derived from a very large set of nuclei. A related novel aspect of the developed model is that we consider four distinct epigenetic classes of TADs as four corresponding types of beads, as well as two additional bead types - pericentromeric constitutive heterochromatin (HET) and centromeric regions (CEN) beads.

The second key methodological novelty of our modeling approach is that we were able to simulate temporal evolution of all the chromosomes in the nucleus on the time-scale of the entire interphase. That a computer simulation of a fruit fly nucleus can reach the biological time-scales of $11 \mathrm{hrs}$ or so is not entirely obvious. Here, we have successfully adapted to the field of chromatin simulations the approach developed in Ref. [91] - implicit solvation with low Langevin friction term. This approach has been proved to be very successful in the study of protein folding and similar problems in structural biology that faced the same problem: biologically interesting time-scales are far out of reach of traditional simulation techniques often used in this field. While we can not map the resulting time-scales precisely onto real biological time, we are confident that what we call "minutes" in our simulations are not hours or seconds of real biological time (see Fig. S3 in the SI).

To arrive at the key interaction parameters that define the "behavior" of the model, we have used three major criteria, or "rules": (1) maximizing Pearson's correlation coefficient between model derived TAD-TAD contact probability map and the experimental Hi-C map; (2) a match between the model derived and experimental set-averaged fractions of LADs near the NE; (3) the conditions for the chromatin A/B compartmentalization - the Flory-Huggins phase separation criterion [97]. The criterion (2) is likely a novelty in development of coarse-grained models of chromatin, which leads to important biological conclusions, see below.

The chromosomal contact Hi-C map derived from the model reproduces all the main qualitative features of the experimental Hi-C maps obtained from Drosophila cells $[1,43]$. These include increased interactions within chromosomal arms, longrange chromatin contacts visible as bright spots located off the main diagonal, genome compartmentalization manifested as plaid-patterns of TAD-TAD contacts, and the Rabl-like configuration represented by interactions between chromosome arms as "wings" stretched perpendicular to the main diagonal.

As an independent validation of the model, it reproduces the key qualitative result from Ref. [44] that lamin depletion enhances interactions between active and inactive chromatin. In another independent validation of the model, we have demonstrated that it reproduces, automatically, experimental chromatin density profiles of both the WT and Lamin mutant nuclei, including some highly nuanced features such the "flatness" of the of density profile away from the NE, Fig. 4, as opposed to, e.g., continued growth of the density toward the center of the nucleus that can be seen when the model parameters deviate from their optimal values, Fig. 6. The fact that the model can reproduce this nuanced behavior is non-trivial, as small 
variations of the model parameters destroy the agreement (while still predicting the more trivial behavior of the chromatin moving away from the NE in the lamina depleted nuclei). Experimentally observed detachment of several individual TADs from the NE in the Lamin mutant [44] is also faithfully reproduced by the model.

\section{Biological predictions}

Our first noteworthy, and likely novel, conclusion is that the positioning of LADs in fruit fly nucleus is highly dynamic (mobile) - on the time scale of the interphase, the same LAD can attach, detach, and re-attach itself to the NE multiple times. This conclusion is supported by the analysis of the distributions of radial positions of single L-TADs: the distributions have two modes - bound and diffusive. Consequently, none of the L-TADs spends all of the time at the NE. This conclusion goes beyond what is known from experiment: that LADs found at the NE differ from cell to cell. What is shown here is that, in any given cell nucleus, LADs are highly dynamic. We argue that this prediction is robust, as it is an inevitable consequence of the relatively low strength of the LAD-NE attraction. The specific value, $4 k T$, of this attractive energy used by our model is not arbitrary, it is derived from the experimental fact that only a specific, limited fraction of LADs are bound to the NE [31]. A hypothetically much higher value of the LAD-NE interaction that could potentially "glue" all or most LADs to the NE would be inconsistent with the experimental data used to construct the model. The observation of the dynamic nature of LADs in interphase nucleus raises a question of the effect of a LADs being in close proximity to the NE on gene expression. More specifically, does the expression level varies as a LAD moves between the periphery and the other parts of the nuclear interior? A recent study systematically tested promoters moved from their native LAD location to a more neutral chromatin environment and to a wide range of chromatin contexts inside LADs. The study has demonstrated that features encoded in the promoter sequence and variation in local chromatin composition determine the gene expression levels in LADs [102]. If the interplay between promoter sequence and local chromatin features is sufficient to determine the level of transcription inside LADs, then gene expression may be robust to the dynamic nature of LADs at least in WT nuclei. Future genome-wide studies of the spatial-temporal transcription inside the nucleus may answer this question; combining experiment and computation may be beneficial.

A related observation about the dynamic LAD binding to the NE is that, despite all of the L-TADs having exactly the same affinity to the NE in our model, the probability of L-TAD binding to the NE turn out to be very different. We explain these differences by pronounced variations, up to 9 times, of the local linear LAD density along the chromatin chains, in contrast to an earlier suggestion that its is highly variable LAD-NE affinities that may be responsible for the differences in the frequency of LAD binding to the NE [40]. A potentially biologically relevant consequence of this observation is that the genetic/epigenetic features of a given TAD alone can not fully determine its fate with respect to probability of being found near the NE, even if the stochastic component of the positioning is eliminated by averaging over time and an ensemble of nuclei. The distribution of LADs along the genome affects the average radial positioning of individual TADs, playing a notable 
role in maintaining a non-random average global structure of chromatin, within its over-all liquid-like state.

The specific strength of the WT value of LAD-NE attraction puts the chromatin very near the "phase boundary" separating two qualitatively different chromatin density distributions: a mere $12 \%(0.5 k T)$ decrease of the LAD-NE affinity strength changes the shape of the chromatin density profile appreciably, from the WT one to one that resembles the Lamin mutant density profile. Changing the LAD-NE affinity by $25 \%$ from its WT value (1 $k T$ decrease) results in a drastic $(60 \%)$ decrease in the fraction of L-TADs at the NE. One proposed biological consequence of being on the "phase boundary" is as follows. If we assume that the $\sim 12 \%(0.5 k T)$ variation in LAD-NE affinity occurs naturally, then the high sensitivity of the chromatin structure to the strength of the LAD-NE affinity might explain variability of chromatin architecture between nuclei of the same tissue and between different tissues. Indeed, some LADs are conserved between cell types, while others are more variable [103]. LADs that display less consistency between cells in a population and tend to be specific to cells where genes located in these LADs are transcriptionally repressed [104]. Cell type-specific genes located in variable LADs are released from NE upon cell type differentiation [38].

Another set of predictions focuses on the potential role of LAD-NE interactions on sensitivity of chromatin 3D architecture to other key interactions (TAD-TAD), which together create the delicate balance that determines the nuclear architecture. Recent works [19, 105, 45, 56, 41], including this one, leave little room to debate the importance of LAD-NE interactions in genome organization. As in previous works, e.g., on mouse [45], agreement of the polymer model with experiment can only be achieved in a rather narrow window of parameters that determine TAD-TAD and LAD-NE interactions in fruit fly nucleus. Our model allows us to differentiate between the four main types of TADs: we find that among transcriptionally repressed TAD types, Null-Null interactions have the strongest effect on the 3D chromatin architecture. Also, as previously reported, one must assume a relatively weak mutual attraction between Active-type TADs. The fact that very different models applied to very different organisms, from fruit fly to mammals [45, 41], arrive at several similar general conclusions regarding the role of the interplay of the interactions between chromatin units and the NE, speaks for a certain degree of conservation of chromatin organization across species.

In contrast to the strong effect of the small changes in LAD-NE interactions on the radial chromatin distribution, the distribution with the WT parameters is rather insensitive to even relatively large (30-100\%) changes in the strength of the interactions between TADs. The changes in the interactions between the Null TADs have the most effect, but even that effect is significantly smaller than the changes in chromatin density profile resulting from similar relative changes in LAD-NE affinity.

Critically, in contrast to WT, chromatin density distribution in the Lamin mutant is extremely sensitive to increase of the strength of cross-type TAD-TAD attractive interactions. This comparison suggests that a role of relatively strong LAD-NE interactions is in providing a stable global environment with a low sensitivity to small fluctuations in TAD-TAD interactions. We speculate that the low sensitivity 
of global chromatin architecture (radial chromatin density distribution) to TADTAD interactions hints at the possibility of a mechanism in which changes in TADTAD interactions can be more important for local regulation of gene transcription activity. We also make a testable prediction that many aspects of the chromatin architecture will be more variable among cells with fewer LAD-NE contacts and even more so in the Lamin-depleted cells. These may include TAD-TAD contacts, chromatin radial distribution, Rabl configuration, spatial segregation of chromosome territories. In fact, a greater variability of the chromatin radial distribution in proventriculus nuclei of the Drosophila Lamin mutant in comparison to WT can be observed by comparing three experimental groups (see Figure S3 in Ref. [88]). Thus, a dramatic loss or dysfunction of lamins during aging or disease [38] may contribute to the increased disorder in gene expression due to greater variability of the global chromatin architecture predicted in this work.

Turning off LAD-NE interactions (simulating Lamin mutant) results in almost complete detachment of the chromatin from the NE and its compaction, causing a 2 -fold increase of the chromatin density in the central region of the nucleus. At the same time, complete removal of the NE results in chromatin decompaction and separation of the chromosome territories on very short time-scale, orders of magnitude shorter that the duration of the interphase. This result suggests that, without the NE, the interactions between TADs are not strong enough to keep the chromatin of the Lamin mutant in a globule-like form on biologically relevant time-scales of hours. At the onset of mitosis, the NE is disassembled and the entire genome is condensed into mitotic chromosomes [106]. In organisms with an open mitosis, such as Drosophila (except syncytial embryonic divisions), NE reformation occurs by recruitment of nuclear pore complexes and membrane components to the surface of the segregating chromosomes. The process begins in late anaphase with the binding of nuclear pore complex proteins to chromosomes and is completed with the recruitment and fusion of membranes during telophase [107]. Thus, the enclosing role of the NE is already established at the beginning of the interphase. This process could have evolved to prevent chromosomes from further unfolding and detaching from each other.

Taken together, these observations suggest a dual mechanical role of the NE in the WT nuclei: it is not simply a confinement of the chromatin, but, rather, the NE acts as an "attractive enclosure" which, at the same time, expands, confines and stabilizes the 3D structure of the chromatin.

Contrary to the chromatin density profiles, which reflect the global 3D chromatin architecture, the predicted Hi-C maps are not very sensitive to the changes in the LAD-NE affinity, suggesting that local chromatin structure is determined mostly by the TAD-TAD interactions. Increasing the interactions strength between the most numerous strongly interacting Null TADs by $1 k T$, from their $1.5 k T$ optimum WT value, leads to a substantial increase in the number of intra-arm and inter-arm contacts. A similar effect on Hi-C map is predicted for the change in interactions between TADs of different types.

By and large, the predicted Lamin mutant Hi-C map looks similar to that of the WT. The differences between the two are mainly in the enhanced inter-chromosome contacts in the mutant, compared to the WT. Small areas where the contact frequency decreases in the mutant are limited to close to diagonal intra-arm contacts. 
These findings are consistent with predictions made for polytene chromosomes in fruit fly [58].

The predicted increase of TAD-TAD contacts and compaction of the chromatin in the Lamin mutant agree with experimental findings. In the Drosophila LamA25 Lamin mutant, the chromatin density is redistributed away from the NE toward the nuclear center [88]. This may bring TADs of different chromosomes closer to each other facilitating inter-chromosome interactions, which is what we observe in simulation. In addition, upon Lamin knockdown in the D. melanogaster S2 cell line [44], overall interaction frequency of chromatin increases. In particular, the Lamin knockdown increases the chromatin density in a fraction of TADs enriched in active chromatin and enhances interactions between active and inactive chromatin [44]. Using our model derived Hi-C maps, we have estimated the sums of contact probabilities of each Null TAD (inactive B-type TADs) with the Active TADs in the Lamin mutant and WT model nuclei, see Fig. S9 in the SI. We found a noticeable, $22 \%$ on average, increase of these active-inactive chromatin contacts in the Lamin depleted nuclei.

Thus, our modeling data indicate that LAD-NE interactions play a prominent role in $3 \mathrm{D}$ genome organization.

\section{Conclusions}

We have developed a coarse-grained model at TAD $(\sim 100 \mathrm{~Kb})$ resolution that describes time evolution of all fruit fly chromosomes, and their interactions with the NE, on the time-scale of the entire interphase. Several methodological novelties proved important to achieve good agreement with experiment, including explicitly accounting for different experimentally observed mutual spatial arrangements of the chromosomes. The model has been verified against multiple experiments, not used in the fitting of its parameters.

We have used the model to explore, in detail, how several key characteristics of the chromatin 3D architecture, including the over-all density distribution and Hi-C map, are sensitive to interaction strength between different classes of TADs, and of the NE with LADs. Some of our general conclusions agree with previous models of mammalian nuclei, that supports conservation of several general principles of chromatin organization across species. Multiple genome-wide predictions have been made, including high mobility of LADs in the interphase, and increased sensitivity of global chromatin architecture to TAD-TAD interactions in lamin depleted nuclei.

We conjecture that one important role of LADs and their attractive interactions with the NE is to create the average global chromatin structure, and to protect its integrity and stability against inevitable cell-to-cell variations of TAD-TAD interactions. We also predict greater variability of the chromatin architecture due to loss or dysfunction of lamins, which may contribute to the increased disorder in gene expression during aging or disease.

\section{Abbreviations}

3D: three-dimensional; CEN: centromeric; Chr: chromosome; HET: pericentromeric constitutive heterochromatin; LAD: lamina-associated domain; L-TAD: TAD that contains LADs; LJ: Lennard-Jones; MSD: mean squared displacement; NE: nuclear envelope; TAD: topologically associating domain; WT: wild-type. 
bioRxiv preprint doi: https://doi.org/10.1101/2022.01.28.478236; this version posted January 28, 2022. The copyright holder for this preprint (which was not certified by peer review) is the author/funder, who has granted bioRxiv a license to display the preprint in perpetuity. It is made

\title{
Declarations
}

\section{Acknowledgements}

We thank Raju Nadimpalli for help with ESPResSO input scripts, chromatin visualization and Hi-C map processing; Samira Mali for technical assistance and for producing the movie clip of the chromosomes dynamics; Yoonjin Kim for technical assistance; Frank Alber for a useful discussion and for providing a comprehensive set of configurational snapshots from Ref. [19].

\section{Funding}

This study was supported by the National Science Foundation [MCB-1715207]

\section{Authors' contributions}

IT and AO designed the dynamical model with input from IS and NK. IT implemented the model with early input from NK. IT, AO and IS analyzed the results. IT, AO, IS and NK wrote the manuscript.

\begin{abstract}
Availability of data and material
The modeling code ESPResSo 3.3.1 [89] used in this research is available at http://espressomd.org/. The software is free, open-source, published under the GNU General Public License (GPL3). The computational protocols for coarse-grained MD simulations and data analysis, as well as data files are available from the authors upon request. Visualization of motion of randomly selected L-TADs in each chromosome on a time-scale of 20 minute is available at https://www.dropbox.com/sh/11r65jvanyua0re/AAA3QkiS3Ry0BIla34sCfrtJa?dl=0
\end{abstract}

Ethics approval and consent to participate

Not applicable.

Competing interests

The authors declare that they have no competing interests.

Consent for publication

All authors read and approved the manuscript.

\section{Author details}

${ }^{1}$ Department of Computer Science, Virginia Tech, 24061, Blacksburg, VA, USA. ${ }^{2}$ Department of Entomology, Virginia Tech, 24061, Blacksburg, VA, USA. ${ }^{3}$ Edward Via College of Osteopathic Medicine, 2265 Kraft Drive, 24060, Blacksburg, VA, USA. ${ }^{4}$ Department of Physics, Virginia Tech, 24061, Blacksburg, VA, USA. ${ }^{5}$ Center for Soft Matter and Biological Physics, Virginia Tech, 24061, Blacksburg, VA, USA.

\section{References}

1. Sexton, T., Yaffe, E., Kenigsberg, E., Bantignies, F., Leblanc, B., Hoichman, M., Parrinello, H., Tanay, A., Cavalli, G.: Three-dimensional folding and functional organization principles of the drosophila genome. Cell 148(3), 458-472 (2012)

2. Bonev, B., Cavalli, G.: Organization and function of the $3 d$ genome. Nature Reviews Genetics 17(11) 661-678 (2016). doi:10.1038/nrg.2016.112

3. Ciabrelli, F., Cavalli, G.: Chromatin-driven behavior of topologically associating domains. J Mol Biol 427(3), 608-25 (2015). doi:10.1016/j.jmb.2014.09.013

4. Barton, L.J., Soshnev, A.A., Geyer, P.K.: Networking in the nucleus: a spotlight on lem-domain proteins. Curr Opin Cell Biol 34, 1-8 (2015). doi:10.1016/j.ceb.2015.03.005

5. Lemaître, C., Bickmore, W.A.: Chromatin at the nuclear periphery and the regulation of genome functions. Histochemistry and Cell Biology 144(2), 111-122 (2015). doi:10.1007/s00418-015-1346-y

6. Nguyen, H.Q., Bosco, G.: Gene positioning effects on expression in eukaryotes. Annu Rev Genet 49, 627-46 (2015). doi:10.1146/annurev-genet-112414-055008

7. Sexton, T., Cavalli, G.: The role of chromosome domains in shaping the functional genome. Cell 160(6), 1049-1059 (2015). doi:10.1016/j.cell.2015.02.040

8. Wang, Y., Nagarajan, M., Uhler, C., Shivashankar, G.V.: Orientation and repositioning of chromosomes correlate with cell geometry-dependent gene expression. Molecular Biology of the Cell 28(14), 1997-2009 (2017). doi:10.1091/mbc.e16-12-0825

9. Diament, A., Tuller, T.: Modeling three-dimensional genomic organization in evolution and pathogenesis. Seminars in Cell \& Developmental Biology 90, 78-93 (2019). doi:10.1016/j.semcdb.2018.07.008

10. Acemel, R.D., Maeso, I., Gómez-Skarmeta, J.L.: Topologically associated domains: a successful scaffold for the evolution of gene regulation in animals. Wiley Interdisciplinary Reviews: Developmental Biology 6(3), 265 (2017)

11. Szabo, Q., Bantignies, F., Cavalli, G.: Principles of genome folding into topologically associating domains. Science Advances 5(4) (2019). doi:10.1126/sciadv.aaw1668. https://advances.sciencemag.org/content/5/4/eaaw1668.full.pdf

12. Wang, S., Su, J.-H., Beliveau, B.J., Bintu, B., Moffitt, J.R., Wu, C.-t., Zhuang, X.: Spatial organization of chromatin domains and compartments in single chromosomes. Science 353(6299), 598-602 (2016)

13. Dixon, J.R., Selvaraj, S., Yue, F., Kim, A., Li, Y., Shen, Y., Hu, M., Liu, J.S., Ren, B.: Topological domains in mammalian genomes identified by analysis of chromatin interactions. Nature 485(7398), 376-380 (2012)

14. Phillips-Cremins, J.E., Sauria, M.E., Sanyal, A., Gerasimova, T.I., Lajoie, B.R., Bell, J.S., Ong, C.-T., Hookway, T.A., Guo, C., Sun, Y., et al.: Architectural protein subclasses shape 3d organization of genomes during lineage commitment. Cell 153(6), 1281-1295 (2013)

15. Rao, S.S., Huntley, M.H., Durand, N.C., Stamenova, E.K., Bochkov, I.D., Robinson, J.T., Sanborn, A.L., Machol, I., Omer, A.D., Lander, E.S., et al.: A 3d map of the human genome at kilobase resolution reveals principles of chromatin looping. Cell 159(7), 1665-1680 (2014) 
bioRxiv preprint doi: https://doi.org/10.1101/2022.01.28.478236; this version posted January 28, 2022. The copyright holder for this preprint (which was not certified by peer review) is the author/funder, who has granted bioRxiv a license to display the preprint in perpetuity. It is made

Tolokh et al. available under aCC-BY-NC 4.0 International license.

Page 23 of 34

16. Dixon, J.R., Jung, I., Selvaraj, S., Shen, Y., Antosiewicz-Bourget, J.E., Lee, A.Y., Ye, Z., Kim, A., Rajagopal, N., Xie, W., et al.: Chromatin architecture reorganization during stem cell differentiation. Nature 518(7539), 331-336 (2015)

17. Renschler, G., Richard, G., Valsecchi, C.I.K., Toscano, S., Arrigoni, L., Ramírez, F., Akhtar, A.: Hi-c guided assemblies reveal conserved regulatory topologies on $\mathrm{x}$ and autosomes despite extensive genome shuffling. Genes \& development 33(21-22), 1591-1612 (2019)

18. Torosin, N.S., Anand, A., Golla, T.R., Cao, W., Ellison, C.E.: 3d genome evolution and reorganization in the drosophila melanogaster species group. bioRxiv (2020)

19. Li, Q., Tjong, H., Li, X., Gong, K., Zhou, X.J., Chiolo, I., Alber, F.: The three-dimensional genome organization of drosophila melanogaster through data integration. Genome biology 18(1), 145-166 (2017)

20. Hua, N., Tjong, H., Shin, H., Gong, K., Zhou, X.J., Alber, F.: Producing genome structure populations with the dynamic and automated pgs software. Nature Protocols 13(5), 915-926 (2018) doi:10.1038/nprot.2018.008

21. Lieberman-Aiden, E., Van Berkum, N.L., Williams, L., Imakaev, M., Ragoczy, T., Telling, A., Amit, I., Lajoie B.R., Sabo, P.J., Dorschner, M.O., et al.: Comprehensive mapping of long-range interactions reveals folding principles of the human genome. science 326(5950), 289-293 (2009)

22. Nora, E.P., Lajoie, B.R., Schulz, E.G., Giorgetti, L., Okamoto, I., Servant, N., Piolot, T., van Berkum, N.L., Meisig, J., Sedat, J., et al:: Spatial partitioning of the regulatory landscape of the x-inactivation centre. Nature 485(7398), 381-385 (2012)

23. Spector, D.L.: The dynamics of chromosome organization and gene regulation. Annual Review of Biochemistry 72(1), 573-608 (2003). doi:10.1146/annurev.biochem.72.121801.161724

24. Lanctôt, C., Cheutin, T., Cremer, M., Cavalli, G., Cremer, T.: Dynamic genome architecture in the nuclear space: regulation of gene expression in three dimensions. Nature Reviews Genetics 8(2), 104-115 (2007). doi: $10.1038 / \mathrm{nrg} 2041$

25. Chubb, J.R., Boyle, S., Perry, P., Bickmore, W.A.: Chromatin motion is constrained by association with nuclear compartments in human cells. Current Biology 12(6), 439-445 (2002). doi:10.1016/s0960-9822(02)00695-4

26. Csink, A.K., Henikoff, S.: Large-scale chromosomal movements during interphase progression in drosophila. Journal of Cell Biology 143(1), 13-22 (1998). doi:10.1083/jcb.143.1.13. https://rupress.org/jcb/article-pdf/143/1/13/1278996/9804005.pdf

27. Spichal, M., Fabre, E.: The emerging role of the cytoskeleton in chromosome dynamics. Frontiers in Genetics 8, 60 (2017). doi:10.3389/fgene. 2017.00060

28. Marshall, W.F.: Order and disorder in the nucleus. Current Biology 12(5), 185-192 (2002). doi:10.1016/S0960-9822(02)00724-8

29. Bronshtein, I., Kepten, E., Kanter, I., Berezin, S., Lindner, M., Redwood, A.B., Mai, S., Gonzalo, S., Foisner, R., Shav-Tal, Y., Garini, Y.: Loss of lamin a function increases chromatin dynamics in the nuclear interior Nature Communications 6(1), 8044 (2015). doi:10.1038/ncomms9044

30. Caridi, C.P., D'Agostino, C., Ryu, T., Zapotoczny, G., Delabaere, L., Li, X., Khodaverdian, V.Y., Amaral, N., Lin, E., Rau, A.R., et al.: Nuclear f-actin and myosins drive relocalization of heterochromatic breaks. Nature 559(7712), 54-60 (2018). doi:10.1038/s41586-018-0242-8

31. Pickersgill, H., Kalverda, B., De Wit, E., Talhout, W., Fornerod, M., van Steensel, B.: Characterization of the drosophila melanogaster genome at the nuclear lamina. Nature genetics 38(9), 1005-1014 (2006)

32. Guelen, L., Pagie, L., Brasset, E., Meuleman, W., Faza, M.B., Talhout, W., Eussen, B.H., de Klein, A., Wessels, L., de Laat, W., et al.: Domain organization of human chromosomes revealed by mapping of nuclear lamina interactions. Nature 453(7197), 948-951 (2008)

33. Prokocimer, M., Davidovich, M., Nissim-Rafinia, M., Wiesel-Motiuk, N., Bar, D.Z., Barkan, R., Meshorer, E., Gruenbaum, Y.: Nuclear lamins: key regulators of nuclear structure and activities. Journal of cellular and molecular medicine 13(6), 1059-1085 (2009)

34. Peric-Hupkes, D., Meuleman, W., Pagie, L., Bruggeman, S.W., Solovei, I., Brugman, W., Gräf, S., Flicek, P. Kerkhoven, R.M., van Lohuizen, M., et al.: Molecular maps of the reorganization of genome-nuclear lamina interactions during differentiation. Molecular cell 38(4), 603-613 (2010)

35. Shevelyov, Y.Y., Ulianov, S.V.: The nuclear lamina as an organizer of chromosome architecture. Cells 8(2), 136 (2019). doi:10.3390/cells8020136

36. van Bemmel, J.G., Pagie, L., Braunschweig, U., Brugman, W., Meuleman, W., Kerkhoven, R.M., van Steensel, B.: The insulator protein su(hw) fine-tunes nuclear lamina interactions of the drosophila genome. PloS ONE 5(11), 15013 (2010)

37. Steensel, B., Belmont, A.: Lamina-associated domains: Links with chromosome architecture, heterochromatin, and gene repression. Cell 169, 780-791 (2017). doi:10.1016/j.cell.2017.04.022

38. Briand, N., Collas, P.: Lamina-associated domains: peripheral matters and internal affairs. Genome Biology 21(85) (2020)

39. Kind, J., Pagie, L., Ortabozkoyun, H., Boyle, S., de Vries, S.S., Janssen, H., Amendola, M., Nolen, L.D., Bickmore, W.A., van Steensel, B.: Single-cell dynamics of genome-nuclear lamina interactions. Cell 153(1), 178-192 (2013). doi:10.1016/j.cell.2013.02.028

40. Kind, J., Pagie, L., de Vries, S.S., Nahidiazar, L., Dey, S.S., Bienko, M., Zhan, Y., Lajoie, B., de Graaf, C.A., Amendola, M., Fudenberg, G., Imakaev, M., Mirny, L.A., Jalink, K., Dekker, J., van Oudenaarden, A., van Steensel, B.: Genome-wide maps of nuclear lamina interactions in single human cells. Cell 163(1), 134-147 (2015). doi:10.1016/j.cell.2015.08.040

41. Chiang, M., Michieletto, D., Brackley, C.A., Rattanavirotkul, N., Mohammed, H., Marenduzzo, D., Chandra, T.: Polymer modeling predicts chromosome reorganization in senescence. Cell Reports 28(12), 3212-32236 (2019). doi:10.1016/j.celrep.2019.08.045

42. Gonzalez-Sandoval, A., Gasser, S.M.: On tads and lads: spatial control over gene expression. Trends in Genetics 32(8), 485-495 (2016)

43. Hou, C., Li, L., Qin, Z.S., Corces, V.G.: Gene density, transcription, and insulators contribute to the partition 
bioRxiv preprint doi: https://doi.org/10.1101/2022.01.28.478236; this version posted January 28, 2022. The copyright holder for this preprint (which was not certified by peer review) is the author/funder, who has granted bioRxiv a license to display the preprint in perpetuity. It is made available under aCC-BY-NC 4.0 International license.

Tolokh et al.

Page 24 of 34

of the drosophila genome into physical domains. Molecular cell 48(3), 471-484 (2012)

44. Ulianov, S., Doronin, S., Khrameeva, E., Kos, P., Luzhin, A., Starikov, S., Galitsyna, A., Nenasheva, V., Ilyin, A., Flyamer, I., Mikhaleva, E., Logacheva, M., Gelfand, M., Chertovich, A., Gavrilov, A., Razin, S., Shevelyov, Y.: Nuclear lamina integrity is required for proper spatial organization of chromatin in drosophila. Nature Communications 10(1), 1176 (2019). doi:10.1038/s41467-019-09185-y

45. Falk, M., Feodorova, Y., Naumova, N., Imakaev, M., Lajoie, B.R., Leonhardt, H., Joffe, B., Dekker, J., Fudenberg, G., Solovei, I., Mirny, L.A.: Heterochromatin drives compartmentalization of inverted and conventional nuclei. Nature 570(7761), 395-399 (2019). doi:10.1038/s41586-019-1275-3

46. Sati, S., Bonev, B., Szabo, Q., Jost, D., Bensadoun, P., Serra, F., Loubiere, V., Papadopoulos, G.L., Rivera-Mulia, J.-C., Fritsch, L., Bouret, P., Castillo, D., Gelpi, J.L., Orozco, M., Vaillant, C., Pellestor, F., Bantignies, F., Marti-Renom, M.A., Gilbert, D.M., Lemaitre, J.-M., Cavalli, G.: 4d genome rewiring during oncogene-induced and replicative senescence. Molecular cell 78(3), 522-5389 (2020). doi:10.1016/j.molcel.2020.03.007

47. Zhou, R., Gao, Y.Q.: A dna sequence based polymer model for chromatin folding. International Journal of Molecular Sciences 22(3) (2021). doi:10.3390/ijms22031328

48. Brunet, A., Destainville, N., Collas, P.: Physical constraints in polymer modeling of chromatin associations with the nuclear periphery at kilobase scale. Nucleus 12(1), 6-20 (2021). doi:10.1080/19491034.2020.1868105. PMID: 33435761. https://doi.org/10.1080/19491034.2020.1868105

49. Laghmach, R., Di Pierro, M., Potoyan, D.A.: Mesoscale liquid model of chromatin recapitulates nuclear order of eukaryotes. Biophysical Journal 118(9), 2130-2140 (2020). doi:10.1016/j.bpj.2019.09.013

50. Boettiger, A.N., Bintu, B., Moffitt, J.R., Wang, S., Beliveau, B.J., Fudenberg, G., Imakaev, M., Mirny, L.A., Wu, C.-t., Zhuang, X.: Super-resolution imaging reveals distinct chromatin folding for different epigenetic states. Nature 529(7586), 418-422 (2016)

51. MacPherson, Q., Beltran, B., Spakowitz, A.J.: Bottom-up modeling of chromatin segregation due to epigenetic modifications. Proceedings of the National Academy of Sciences 115(50), 12739-12744 (2018). doi:10.1073/pnas.1812268115. https://www.pnas.org/content/115/50/12739.full.pdf

52. Rosa, A., Everaers, R.: Structure and dynamics of interphase chromosomes. PLoS Computational Biology 4(8), 1000153 (2008). doi:10.1371/journal.pcbi.1000153

53. Barbieri, M., Chotalia, M., Fraser, J., Lavitas, L.-M., Dostie, J., Pombo, A., Nicodemi, M.: Complexity of chromatin folding is captured by the strings and binders switch model. Proceedings of the National Academy of Sciences 109(40), 16173-16178 (2012)

54. Sanborn, A., Rao, S., Huang, S.-C., Durand, N., Huntley, M., Jewett, A., Bochkov, I., Chinnappan, D., Cutkosky, A., Li, J., Geeting, K., Gnirke, A., Melnikov, A., McKenna, D., Stamenova, E., Lander, E., Aiden, E.: Chromatin extrusion explains key features of loop and domain formation in wild-type and engineered genomes. Proceedings of the National Academy of Sciences 112(47), 6456-6465 (2015). doi:10.1073/pnas.1518552112, url = http://dx.doi.org/10.1073/pnas.1518552112,

55. Zhang, B., Wolynes, P.G.: Topology, structures, and energy landscapes of human chromosomes. Proceedings of the National Academy of Sciences 112(19), 6062-6067 (2015)

56. Kinney, N.A., Sharakhov, I.V., Onufriev, A.V.: Chromosome-nuclear envelope attachments affect interphase chromosome territories and entanglement. Epigenetics \& Chromatin 11, 3 (2018)

57. Di Pierro, M., Cheng, R.R., Lieberman-Aiden, E., Wolynes, P.G., Onuchic, J.N.: De novo prediction of human chromosome structures: Epigenetic marking patterns encode genome architecture. Proceedings of the National Academy of Sciences 114(46), 12126-12131 (2017)

58. Kinney, N., Onufriev, A., Sharakhov, I.: Quantified effects of chromosome-nuclear envelope attachments on 3d organization of chromosomes. Nucleus 6(3), 212-224 (2015). doi:10.1080/19491034.2015.1056441

59. Tjong, H., Gong, K., Chen, L., Alber, F.: Physical tethering and volume exclusion determine higher-order genome organization in budding yeast. Genome research 22(7), 1295-1305 (2012). doi:10.1101/gr.129437.111

60. Wong, H., Marie-Nelly, H., Herbert, S., Carrivain, P., Blanc, H., Koszul, R., Fabre, E., Zimmer, C.: A predictive computational model of the dynamic 3d interphase yeast nucleus. Current Biology 22(20), 1881-1890 (2012). doi:10.1016/j.cub.2012.07.069

61. Tokuda, N., Terada, T., Sasai, M.: Dynamical modeling of three-dimensional genome organization in interphase budding yeast. Biophysical journal 102(2), 296-304 (2012). doi:10.1016/j.bpj.2011.12.005

62. Jerabek, H., Heermann, D.: How Chromatin Looping and Nuclear Envelope Attachment Affect Genome Organization in Eukaryotic Cell Nuclei, vol. 307, pp. 351-381. Elsevier, ??? (2014). doi:10.1016/b978-0-12-800046-5.00010-2. http://dx.doi.org/10.1016/b978-0-12-800046-5.00010-2

63. Shi, G., Liu, L., Hyeon, C., Thirumalai, D.: Interphase human chromosome exhibits out of equilibrium glassy dynamics. Nature Communications 9(1), 3161 (2018). doi:10.1038/s41467-018-05606-6

64. Bruinsma, R., Grosberg, A., Rabin, Y., Zidovska, A.: Chromatin hydrodynamics. Biophysical Journal 106(9), 1871-1881 (2014). doi:10.1016/j.bpj.2014.03.038

65. Haddad, N., Jost, D., Vaillant, C.: Perspectives: using polymer modeling to understand the formation and function of nuclear compartments. Chromosome Research 25(1), 35-50 (2017)

66. Ghosh, S.K., Jost, D.: How epigenome drives chromatin folding and dynamics, insights from efficient coarse-grained models of chromosomes. PLoS Computational Biology 14(5), 1006159 (2018)

67. Di Stefano, M., Nützmann, H.-W., Marti-Renom, M.A., Jost, D.: Polymer modelling unveils the roles of heterochromatin and nucleolar organizing regions in shaping $3 \mathrm{~d}$ genome organization in arabidopsis thaliana. Nucleic Acids Research 49(4), 1840-1858 (2021). doi:10.1093/nar/gkaa1275. https://academic.oup.com/nar/article-pdf/49/4/1840/36398531/gkaa1275.pdf

68. MacKay, K., Kusalik, A.: Computational methods for predicting 3d genomic organization from high-resolution chromosome conformation capture data. Briefings in functional genomics 19(4), 292-308 (2020)

69. Kalhor, R., Tjong, H., Jayathilaka, N., Alber, F., Chen, L.: Genome architectures revealed by tethered chromosome conformation capture and population-based modeling. Nature biotechnology 30(1), 90-98 (2012) 
bioRxiv preprint doi: https://doi.org/10.1101/2022.01.28.478236; this version posted January 28, 2022. The copyright holder for this preprint (which was not certified by peer review) is the author/funder, who has granted bioRxiv a license to display the preprint in perpetuity. It is made available under aCC-BY-NC 4.0 International license.

Tolokh et al.

Page 25 of 34

70. Tjong, H., Li, W., Kalhor, R., Dai, C., Hao, S., Gong, K., Zhou, Y., Li, H., Zhou, X.J., Le Gros, M.A., et al.: Population-based 3d genome structure analysis reveals driving forces in spatial genome organization. Proceedings of the National Academy of Sciences 113(12), 1663-1672 (2016)

71. Serra, F., Baù, D., Goodstadt, M., Castillo, D., Filion, G.J., Marti-Renom, M.A.: Automatic analysis and 3d-modelling of hi-c data using tadbit reveals structural features of the fly chromatin colors. PLoS Computational Biology 13(7), 1005665 (2017)

72. Paulsen, J., Ali, T.M.L., Collas, P.: Computational 3d genome modeling using chrom3d. Nature protocols 13(5), 1137-1152 (2018)

73. Ulianov, S.V., Zakharova, V.V., Galitsyna, A.A., Kos, P.I., Polovnikov, K.E., Flyamer, I.M., Mikhaleva, E.A., Khrameeva, E.E., Germini, D., Logacheva, M.D., et al.: Order and stochasticity in the folding of individual drosophila genomes. Nature Communications 12(1), 41 (2021). doi:10.1038/s41467-020-20292-z

74. Vivante, A., Brozgol, E., Bronshtein, I., Levi, V., Garini, Y.: Chromatin dynamics governed by a set of nuclear structural proteins. Genes, Chromosomes and Cancer 58(7), 437-451 (2019). doi:10.1002/gcc.22719

75. Maass, P.G., Barutcu, A.R., Weiner, C.L., Rinn, J.L.: Inter-chromosomal contact properties in live-cell imaging and in hi-c. Molecular cell 69(6), 1039-1045 (2018)

76. Shinkai, S., Nakagawa, M., Sugawara, T., Togashi, Y., Ochiai, H., Nakato, R., Taniguchi, Y., Onami, S.: Phi-c: deciphering hi-c data into polymer dynamics. NAR Genomics and Bioinformatics 2(2), 020 (2020)

77. Feig, M., Sugita, Y.: Whole-cell models and simulations in molecular detail. Annual Review of Cell and Developmental Biology 35(1), 191-211 (2019). doi:10.1146/annurev-cellbio-100617-062542. PMID: 31299173. https://doi.org/10.1146/annurev-cellbio-100617-062542

78. Brackley, C.A., Johnson, J., Kelly, S., Cook, P.R., Marenduzzo, D.: Simulated binding of transcription factors to active and inactive regions folds human chromosomes into loops, rosettes and topological domains. Nucleic acids research 44(8), 3503-3512 (2016)

79. Fudenberg, G., Imakaev, M., Lu, C., Goloborodko, A., Abdennur, N., Mirny, L.A.: Formation of chromosomal domains by loop extrusion. Cell reports 15(9), 2038-2049 (2016)

80. Lifshitz, I.M., Grosberg, A.Y., Khokhlov, A.R.: Some problems of the statistical physics of polymer chains with volume interaction. Rev. Mod. Phys. 50, 683-713 (1978). doi:10.1103/RevModPhys.50.683

81. Kremer, K., Grest, G.: Dynamics of entangled linear polymer melts: A molecular-dynamics simulation. The Journal of Chemical Physics 92(8), 5057-5086 (1990). doi:10.1063/1.458541

82. Fung, J.C., Marshall, W.F., Dernburg, A., Agard, D.A., Sedat, J.W., John, A., Sedat, W., Biochemistry, D.O.: Homologous chromosome pairing in drosophila melanogaster proceeds through multiple independent initiations. J. Cell Biol 141, 5-20 (1998)

83. Csink, A.K., Henikoff, S.: Large-scale chromosomal movements during interphase progression in drosophila. The Journal of cell biology 143(1), 13-22 (1998)

84. Child, M., Bateman, J.R., Jahangiri, A., Reimer, A., Lammers, N.C., Sabouni, N., Villamarin, D., Mckenzie-Smith, G.C., Johnson, J.E., Jost, D., Garcia, H.: Live imaging and biophysical modeling support a button-based mechanism of somatic homolog pairing in drosophila. eLife 10, 64412 (2021). doi:10.7554/eLife.64412

85. Abed, J.A., Erceg, J., Goloborodko, A., Nguyen, S.C., Mccole, R.B., Saylor, W., Fudenberg, G., Lajoie, B.R., Dekker, J., Mirny, L.A., Wu, C.-t.: Highly structured homolog pairing reflects functional organization of the drosophila genome. Nature Communications 10, 4485 (2019). doi:10.1038/s41467-019-12208-3

86. Mirny, L.: The fractal globule as a model of chromatin architecture in the cell. Chromosome research 19 , 37-51 (2011). doi:10.1007/s10577-010-9177-0

87. Hochstrasser, M., Mathog, D., Gruenbaum, Y., Saumweber, H., Sedat, J.W.: Spatial organization of chromosomes in the salivary gland nuclei of drosophila melanogaster. The Journal of cell biology 102(1), 112-123 (1986)

88. Bondarenko, S.M., Sharakhov, I.V.: Reorganization of the nuclear architecture in the drosophila melanogaster lamin b mutant lacking the caax box. Nucleus 11(1), 283-298 (2020)

89. Limbach, H.-J., Arnold, A., Mann, B.A., Holm, C.: Espresso-an extensible simulation package for research on soft matter systems. Computer Physics Communications 174(9), 704-727 (2006)

90. Kremer, K., Grest, G.: Molecular dynamics (MD) simulations for polymers. Journal of Physics: Condensed Matter 2(S), 295-298 (1990). doi:10.1088/0953-8984/2/s/045

91. Anandakrishnan, R., Drozdetski, A., Walker, R., Onufriev, A.: Speed of conformational change: Comparing explicit and implicit solvent molecular dynamics simulations. Biophysical Journal 108(5), 1153-1164 (2015). doi:10.1016/j.bpj.2014.12.047

92. Weber, S., Spakowitz, A., Theriot, J.: Bacterial chromosomal loci move subdiffusively through a viscoelastic cytoplasm. Physical review letters 104, 238102 (2010). doi:10.1103/PhysRevLett.104.238102

93. Di Pierro, M., Zhang, B., Aiden, E.L., Wolynes, P.G., Onuchic, J.N.: Transferable model for chromosome architecture. Proceedings of the National Academy of Sciences 113(43), 12168-12173 (2016). doi:10.1073/pnas.1613607113. https://www.pnas.org/content/113/43/12168.full.pdf

94. Collas, P., Liyakat Ali, T.M., Brunet, A., Germier, T.: Finding friends in the crowd: Three-dimensional cliques of topological genomic domains. Frontiers in Genetics 10, 602 (2019). doi:10.3389/fgene.2019.00602

95. Paulsen, J., Ali, T.M.L., Nekrasov, M., Delbarre, E., Baudement, M.-O., Kurscheid, S., Tremethick, D., Collas, P.: Long-range interactions between topologically associating domains shape the four-dimensional genome during differentiation. Nature genetics 51(5), 835-843 (2019)

96. Filion, G., Van Bemmel, J., Braunschweig, U., Talhout, W., Kind, J., Ward, L., Brugman, W., Castro, I., Kerkhoven, R., Bussemaker, H., van Steensel, B.: Systematic protein location mapping reveals five principal chromatin types in drosophila cells. Cell 143, 212-24 (2010). doi:10.1016/j.cell.2010.09.009

97. Flory, P.J.: Thermodynamics of heterogeneous polymers and their solutions. The Journal of Chemical Physics 12(11), 425-438 (1944)

98. Schlosshauer, M., Baker, D.: Realistic protein-protein association rates from a simple diffusional model neglecting long-range interactions, free energy barriers, and landscape ruggedness. Protein Science 13(6), 
bioRxiv preprint doi: https://doi.org/10.1101/2022.01.28.478236; this version posted January 28, 2022. The copyright holder for this preprint (which was not certified by peer review) is the author/funder, who has granted bioRxiv a license to display the preprint in perpetuity. It is made

1660-1669 (2004). doi:10.1110/ps.03517304

99. Avșaroğlu, B., Bronk, G., Gordon-Messer, S., Ham, J., Bressan, D.A., Haber, J.E., Kondev, J.: Effect of chromosome tethering on nuclear organization in yeast. PLOS ONE 9(7), 1-11 (2014). doi:10.1371/journal.pone.0102474

100. Forsberg, F., Brunet, A., Ali, T.M.L., Collas, P.: Interplay of lamin a and lamin b lads on the radial positioning of chromatin. Nucleus 10(1), 7-20 (2019). doi:10.1080/19491034.2019.1570810. PMID: 30663495. https://doi.org/10.1080/19491034.2019.1570810

101. Camps, J., Erdos, M.R., Ried, T.: The role of lamin b1 for the maintenance of nuclear structure and function. Nucleus 6(1), 8-14 (2015). doi:10.1080/19491034.2014.1003510. PMID: 25602590. https://doi.org/10.1080/19491034.2014.1003510

102. Leemans, C., Zwalm, M.V.D., Brueckner, L., Comoglio, F., Schaik, T.V., Pagie, L., Arensbergen, J.V., Steensel, B.V.: Promoter-intrinsic and local chromatin features determine gene repression in lamina-associated domains 177, 852-864 (2018). doi:10.1101/464081

103. Meuleman, W., Peric-Hupkes, D., Kind, J., Beaudry, J.-B., Pagie, L., Kellis, M., Reinders, M., Wessels, L., van Steensel, B.: Constitutive nuclear lamina-genome interactions are highly conserved and associated with a/t-rich sequence. Genome research 23(2), 270-280 (2013)

104. Rullens, P.M.J., Kind, J.: Attach and stretch: Emerging roles for genome-lamina contacts in shaping the 3d genome. Current Opinion in Cell Biology 70, 51-57 (2021). doi:10.1016/j.ceb.2020.11.006. Cell Nucleus

105. Paulsen, J., Sekelja, M., Oldenburg, A.R., Barateau, A., Briand, N., Delbarre, E., Shah, A., Sørensen, A.L., Vigouroux, C., Buendia, B., et al.: Chrom3d: three-dimensional genome modeling from hi-c and nuclear lamin-genome contacts. Genome biology 18(1), 1-15 (2017)

106. Egli, D., Birkhoff, G., Eggan, K.: Mediators of reprogramming: transcription factors and transitions through mitosis. Nature reviews. Molecular cell biolog 9, 505-516 (2008)

107. Güttinger, S., Laurell, E., Kutay, U.: Orchestrating nuclear envelope disassembly and reassembly during mitosis. Nature Reviews Molecular Cell Biology 10(3), 178-191 (2009). doi:10.1038/nrm2641 
bioRxiv preprint doi: https://doi.org/10.1101/2022.01.28.478236; this version posted January 28, 2022. The copyright holder for this preprint (which was not certified by peer review) is the author/funder, who has granted bioRxiv a license to display the preprint in perpetuity. It is made
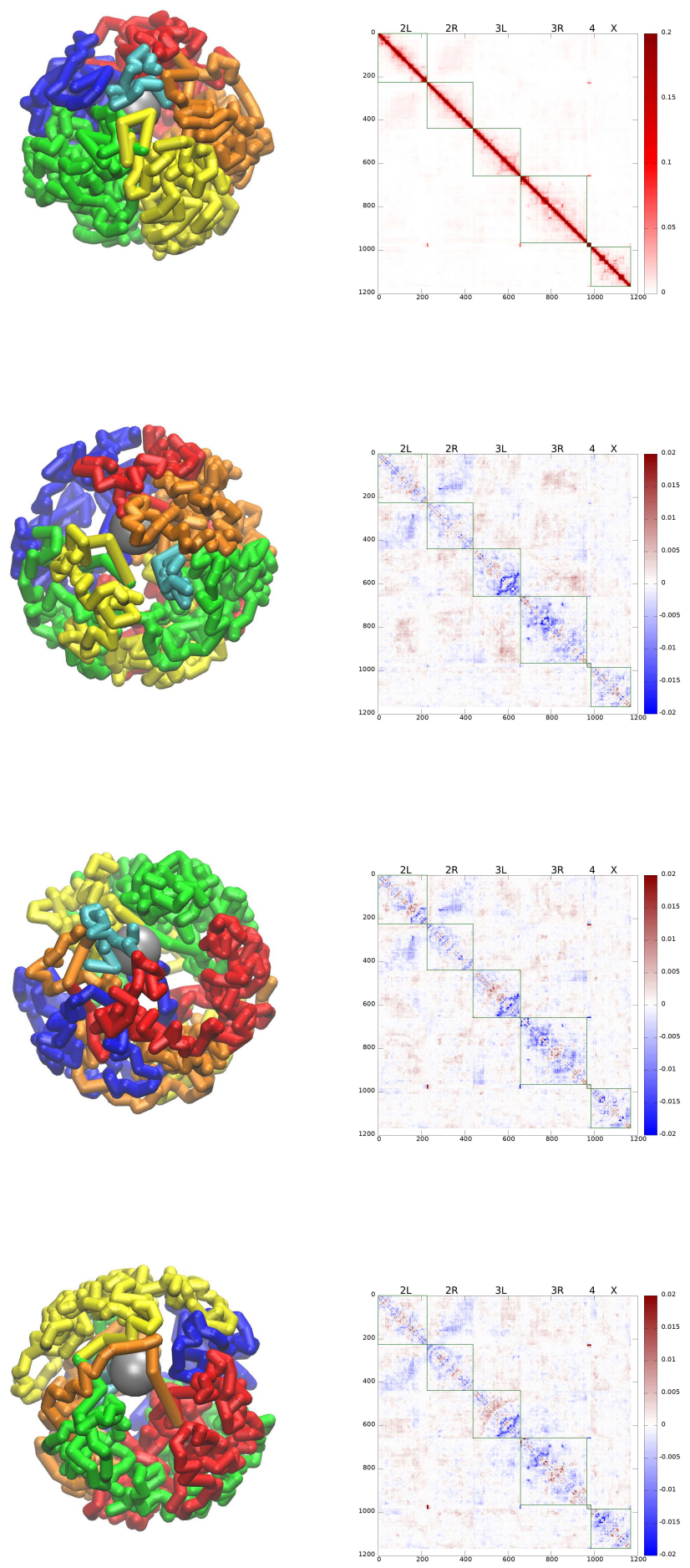

Figure 2 Temporal evolution of the chromatin configurations in the wild-type (WT) nucleus model (left column) and of the model derived TAD-TAD contact probability $(\mathrm{Hi}-\mathrm{C})$ maps (right column). From top to bottom: The chromatin configurations at $t=0 \mathrm{~min}$ (starting configuration), $30 \mathrm{~min}, 3 \mathrm{~h}$ and $11 \mathrm{~h}$. The chromatin configurations shown correspond to TRANS-X3S nucleus chromosome topology (see Fig. 1). The $\mathrm{Hi}-\mathrm{C}$ maps are averaged over 5 min $\left(3 \cdot 10^{6}\right.$ time steps) time intervals and over the ensemble of 18 system trajectories (see "Methods") generated with the WT parameter set. The right most panel in the top row is $\mathrm{t}=0$ min $\mathrm{Hi}-\mathrm{C}$ map, followed (from top to bottom) by the difference maps at $30 \mathrm{~min}, 3 \mathrm{~h}$ and $11 \mathrm{~h}$. 
bioRxiv preprint doi: https://doi.org/10.1101/2022.01.28.478236; this version posted January 28, 2022. The copyright holder for this preprint (which was not certified by peer review) is the author/funder, who has granted bioRxiv a license to display the preprint in perpetuity. It is made
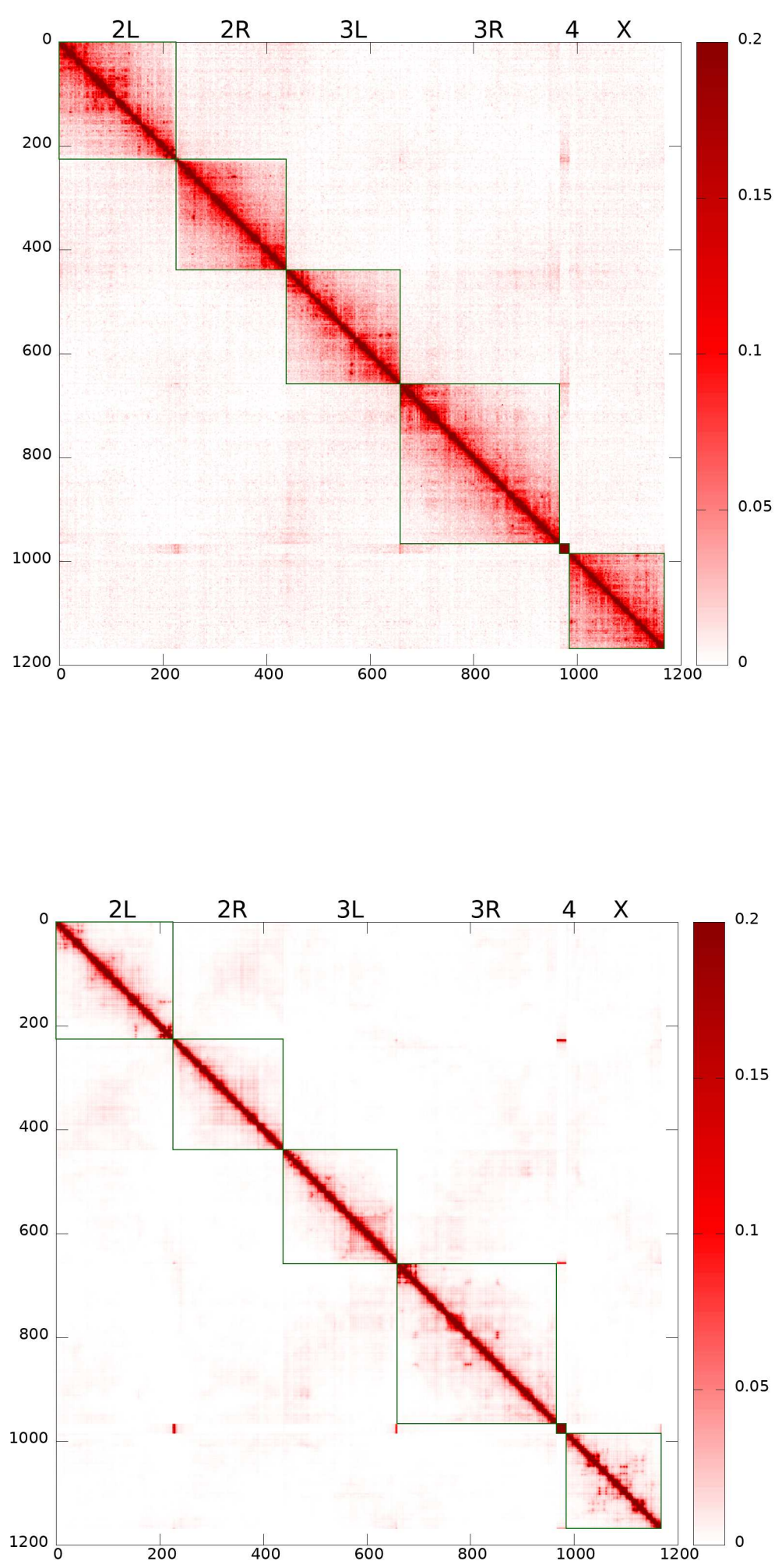

Figure 3 Top panel: Experimental Hi-C map for TAD-TAD contact probabilities in the WT D. melanogaster nuclei [19] (original data for embryonic cells from Ref. [1]). Bottom panel: Model derived TAD-TAD contact probability $(\mathrm{Hi}-\mathrm{C})$ map corresponding to the young $(0-3 \mathrm{~min})$ nuclei. This $\mathrm{Hi}-\mathrm{C}$ map reproduces many features expected from experiment: increased interactions within chromosomal arms, long-range chromatin contacts visible as bright spots, genome compartmentalization manifested as plaid-patterns of TAD-TAD contacts, and the Rabl-like configuration represented by interactions between chromosomal arms (e.g., $2 \mathrm{~L}$ and $2 \mathrm{R}$ ) as "wings" stretched perpendicular to the main diagonal. Pearson's correlation coefficient is 0.956 . 
bioRxiv preprint doi: https://doi.org/10.1101/2022.01.28.478236; this version posted January 28, 2022. The copyright holder for this preprint (which was not certified by peer review) is the author/funder, who has granted bioRxiv a license to display the preprint in perpetuity. It is made Tolokh et al. available under aCC-BY-NC 4.0 International license.

Page 29 of 34
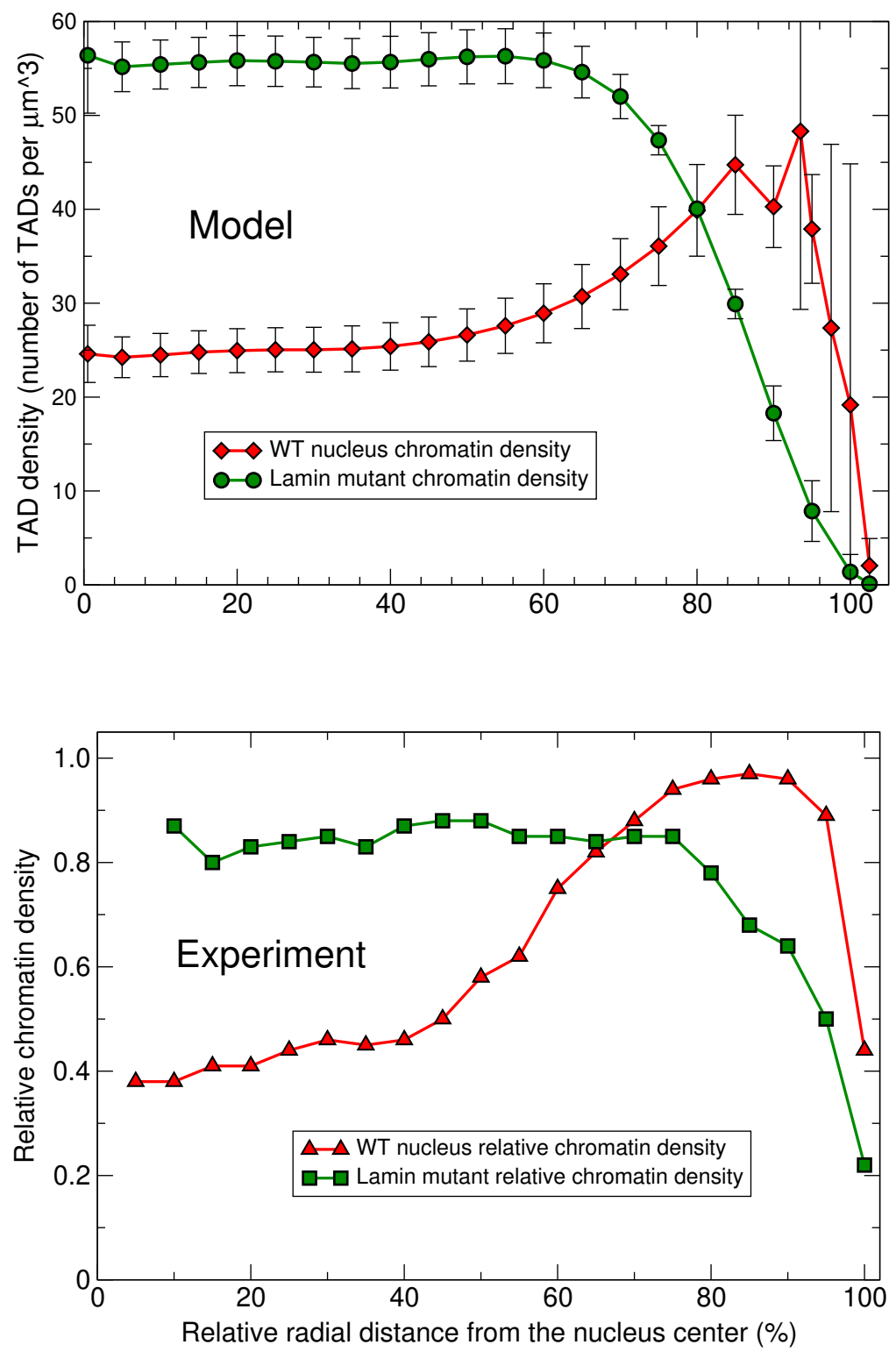

Figure 4 Top panel: Radial chromatin density distributions for the model nuclei with the WT interaction parameter set (red curve, LAD-NE affinity $4 k T$ ) and for the Lamin mutant model nuclei (green curve, LAD-NE affinity $0.1 \mathrm{kT}$ ). Bottom panel: Experimental radial chromatin density distributions corresponding to the WT nuclei (red curve, triangles) and the Lamin mutant nuclei (green curve, squares) [Adapted from Supplementary Data, Figure S3 (Group 1) of [88]]. 
bioRxiv preprint doi: https://doi.org/10.1101/2022.01.28.478236; this version posted January 28, 2022. The copyright holder for this preprint (which was not certified by peer review) is the author/funder, who has granted bioRxiv a license to display the preprint in perpetuity. It is made available under aCC-BY-NC 4.0 International license.
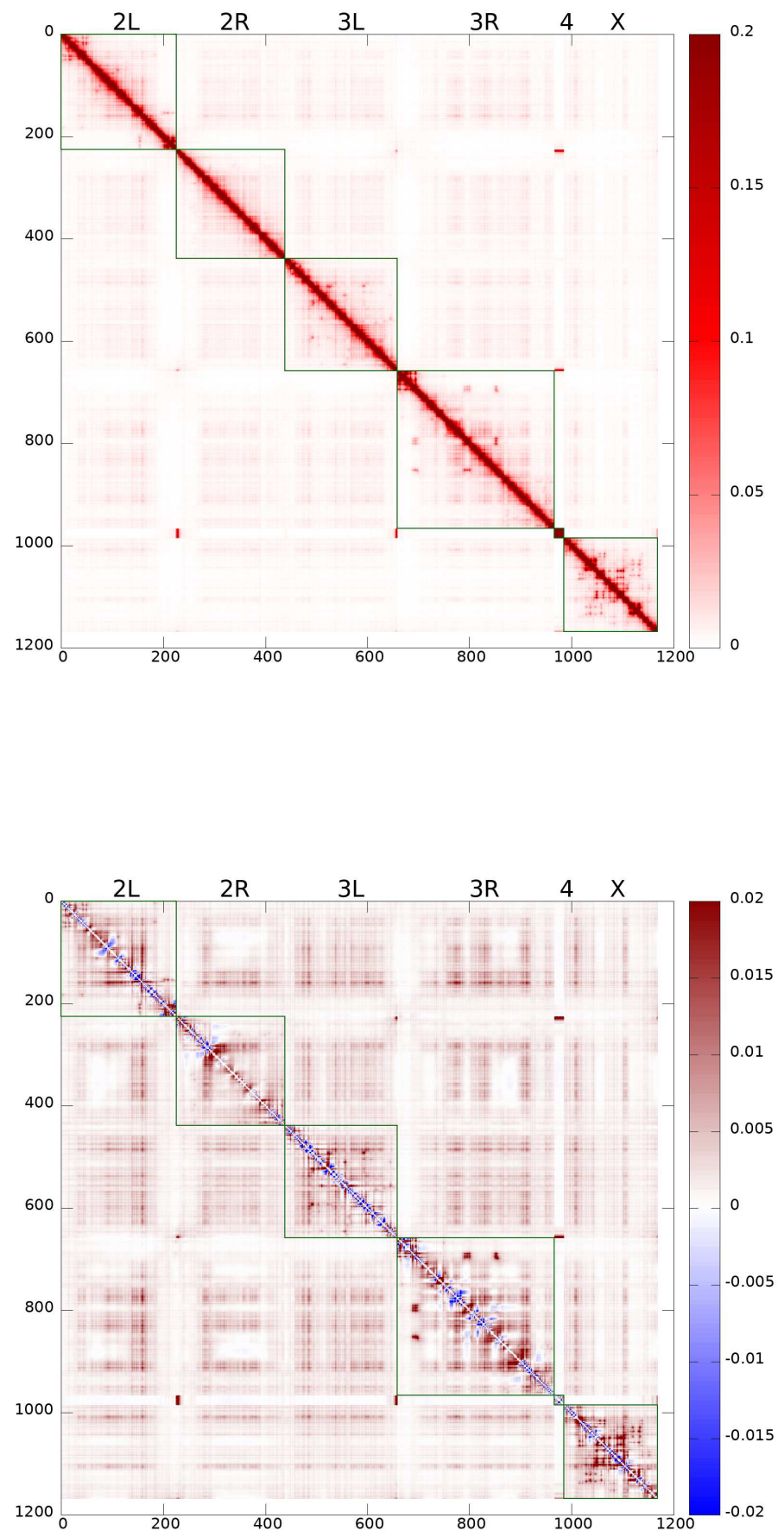

Figure 5 Top panel: Model derived " $11 \mathrm{~h}$ " Lamin mutant TAD-TAD contact probability (HiC) map. Pearson's correlation coefficient relative to the experimental WT Hi-C map is 0.954 , the same as in the case of the "11 h" WT model nuclei, suggesting a high over-all similarity between the WT and mutant contact maps. Bottom panel: Difference between Lamin mutant model $\mathrm{Hi}-\mathrm{C}$ map and WT model Hi-C map. 
bioRxiv preprint doi: https://doi.org/10.1101/2022.01.28.478236; this version posted January 28, 2022. The copyright holder for this preprint (which was not certified by peer review) is the author/funder, who has granted bioRxiv a license to display the preprint in perpetuity. It is made Tolokh et al. available under aCC-BY-NC 4.0 International license.

Page 31 of 34

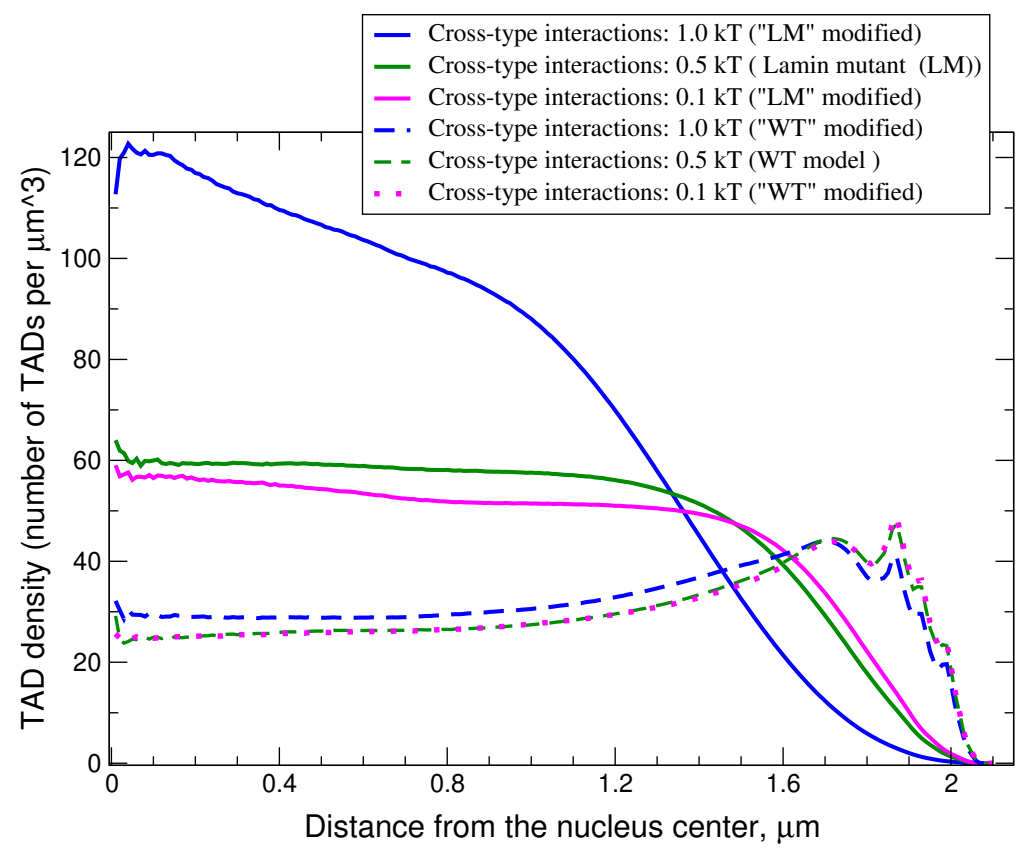

Figure 6 Chromatin density distributions in the nuclei models (derived from the WT and Lamin mutant models) with modified levels of cross-type TAD-TAD attractive interactions (parameter $\epsilon_{A B}$ ), both between $\mathrm{A}$ and $\mathrm{B}$ types of beads and between different types of $\mathrm{B}$ beads (e.g., Null-PcG interaction). 
bioRxiv preprint doi: https://doi.org/10.1101/2022.01.28.478236; this version posted January 28, 2022. The copyright holder for this preprint (which was not certified by peer review) is the author/funder, who has granted bioRxiv a license to display the preprint in perpetuity. It is made Tolokh et al. available under aCC-BY-NC 4.0 International license.

Page 32 of 34

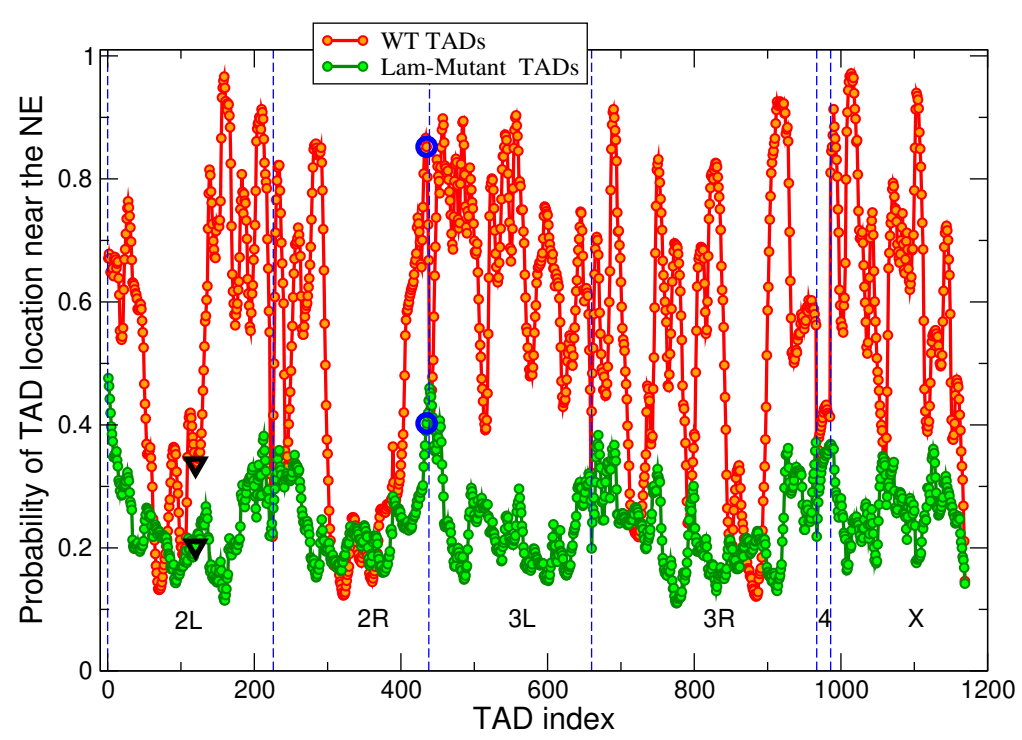

Figure 7 Probabilities of TADs to be within $0.4 \mu \mathrm{m}$ layer adjacent to the NE (half the nuclear volume) for the WT and Lamin mutant model nuclei. Statistical error bars are smaller than symbol size. TADs can be partitioned onto two major groups: the TADs with the probabilities greater than 0.5 , and the TADs with the probabilities in the range 0.1-0.5. The TADs in the second group belong to the sections of chromosomes containing relatively long continuous stretches (10 - 16 TADs) without LADs. PcG L-TAD \#435, analyzed in [44] as cytological region $60 D$, is marked by blue cycles (for the WT and Lamin mutant nuclei). Null L-TADs \#120, described in [44] as cytological region $36 C$, is marked by black triangles. These two regions have been experimentally shown to detach from the NE in the Lamin mutant, providing an additional validation for the model. 
bioRxiv preprint doi: https://doi.org/10.1101/2022.01.28.478236; this version posted January 28, 2022. The copyright holder for this preprint (which was not certified by peer review) is the author/funder, who has granted bioRxiv a license to display the preprint in perpetuity. It is made Tolokh et al. available under aCC-BY-NC 4.0 International license.

Page 33 of 34

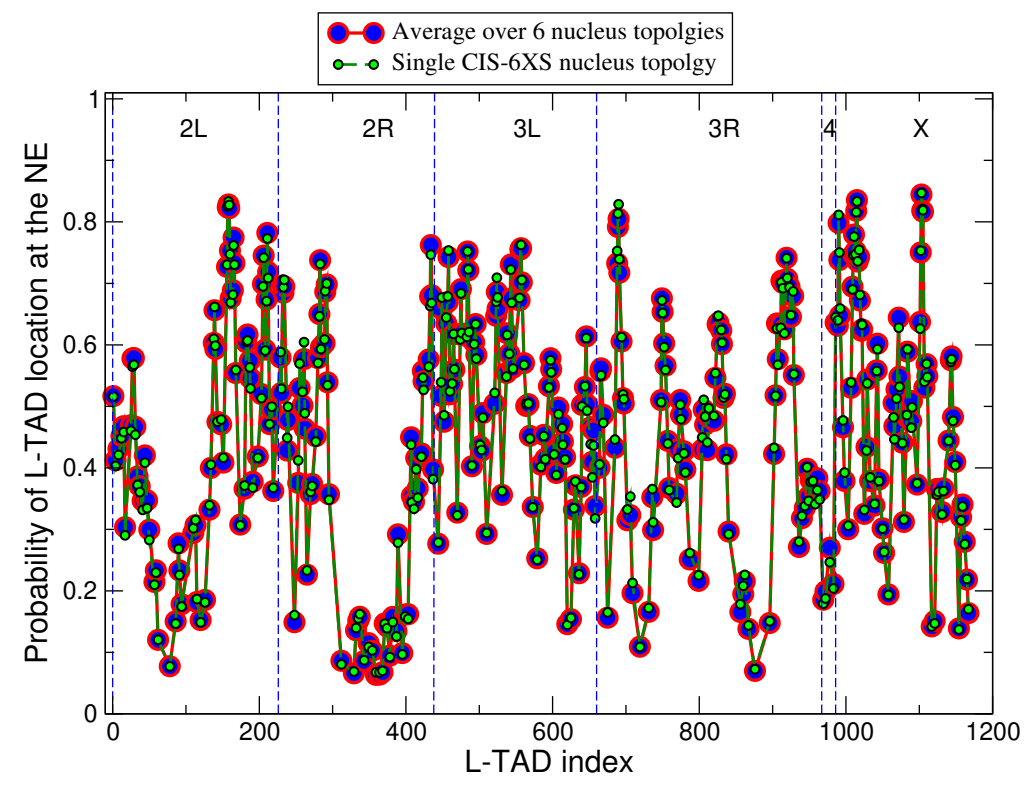

Figure 8 Probabilities of L-TADs (LAD containing TADs) to be to be within $0.2 \mu \mathrm{m}$ layer (average TAD diameter) adjacent to the NE for the WT nuclei. Large red/blue circles are the averages over 6 nuclei with 4 different nucleus topolgies (4 CIS and 2 TRANS). Smaller black/green circles are the probabilities for a single CIS-X6S nucleus topology. All nuclei are of 2 $\mu \mathrm{m}$ radius. Statistical error bars are smaller than symbol size. Despite all $350 \mathrm{~L}-\mathrm{TADs}$ in the model having the same affinity to the NE, there is a significant spread of their probabilities to be near the NE. A non-negligible number of L-TADs, $12 \%$, have a very low (less than 0.2 ) probability to be at the NE. None of the L-TADs stay at the NE all the time, the maximum probability being only 0.85 , suggesting a dynamic nature of all LAD-NE contacts. 
bioRxiv preprint doi: https://doi.org/10.1101/2022.01.28.478236; this version posted January 28, 2022. The copyright holder for this preprint (which was not certified by peer review) is the author/funder, who has granted bioRxiv a license to display the preprint in perpetuity. It is made available under aCC-BY-NC 4.0 International license.
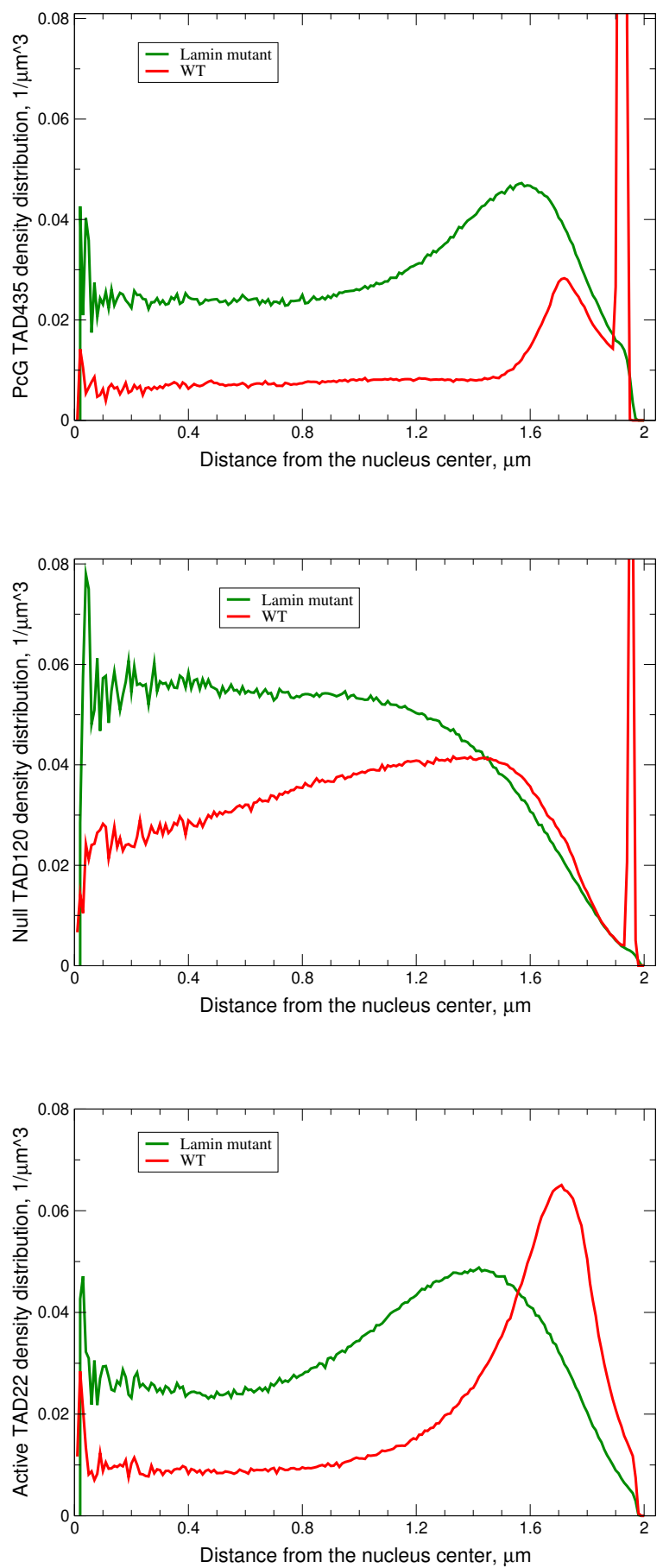

Figure 9 Top panel: Radial distribution of the PcG L-TAD \#435 (TAD $60 D$ in Ref. [44]) in the WT and Lamin mutant nucleus models. Middle panel: Radial distribution of the Null L-TAD \#120 (TAD 36C in Ref. [44]) in the WT and Lamin mutant nuclei. Bottom panel: Radial distribution of the Active TAD \#22 in the WT and Lamin mutant nuclei. The distributions of L-TADs (LADs) are bi-modal: the NE-bound mode is characterized by a high and very narrow density peak right at the NE, and the diffusive mode exhibits a noticeable LAD density in the nuclear interior. The bi-modality is consistent with the mobile nature of LADs. 